\title{
Densidade do conjunto das dinâmicas simbólicas com todas as medidas ergódicas suportadas em órbitas periódicas
}

\author{
Tatiane Cardoso Batista
}

TEse APRESENTADA

$\mathrm{AO}$

Instituto De Matemática e EstatísticA

DA

Universidade De SÃo Paulo

PARA

OBTENÇÃO DO TÍTULO

$\mathrm{DE}$

Doutor EM CIÊNCIAS

Programa: Matemática Aplicada

Orientador: Prof. Dr. Fabio Armando Tal

Durante o desenvolvimento deste trabalho a autora recebeu auxílio financeiro da Capes

São Paulo, agosto de 2013 



\title{
Densidade do conjunto das dinâmicas simbólicas com todas as medidas ergódicas suportadas em órbitas periódicas
}

\author{
Este exemplar corresponde à redação final \\ da tese devidamente corrigida e defendida \\ por Tatiane Cardoso Batista e aprovada \\ pela Comissão Julgadora em 25/10/2013.
}

Banca Examinadora:

- Prof. Dr. Fabio Armando Tal (orientador) - IME-USP.

- Prof. Dr. Rodrigo Bissacot Proença - IME-USP.

- Prof. Dr. Ricardo dos Santos Freire Junior - IME-USP.

- Prof. Dr. Artur Oscar Lopes - UFRGS.

- Prof. Dr. Nestor Felipe Caticha Alfonso - IF-USP. 



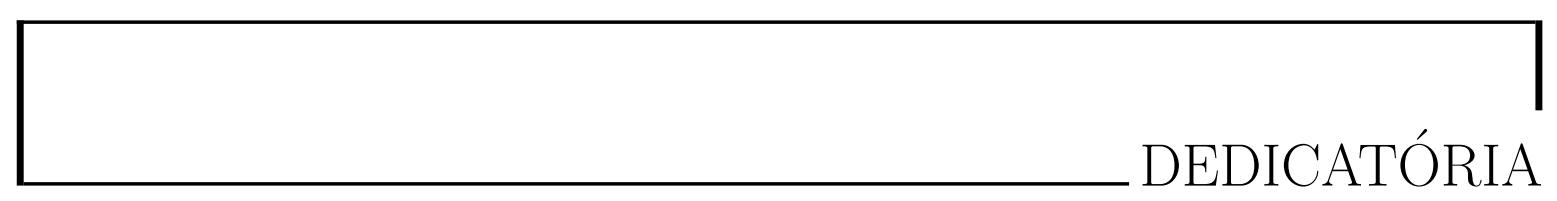

Aos meus pais,

Mauricio e Sinézia. 

Agradeço...

À Deus, sempre presente em minha vida.

Aos meus pais, meu irmão Rodolfo e à toda minha família, pelo carinho que recebo em todos os momentos.

Ao meu noivo Diego, por fazer parte da minha vida e estar sempre ao meu lado.

Ao professor Dr. Fabio Tal, pelo apoio e dedicação na realização desse trabalho.

Ao colega de pesquisa, colega de trabalho e vizinho, Juliano, companheiro de todas as horas!

À todos os meus amigos que fizeram parte dessa caminhada... É muito bom poder contar com vocês!

À Universidade de São Paulo, pela oportunidade de estudo.

À Universidade Tecnológica Federal do Paraná, pelo amparo.

À CAPES, pelo apoio financeiro.

À todos aqueles que contribuíram de alguma maneira...Muito obrigada! 



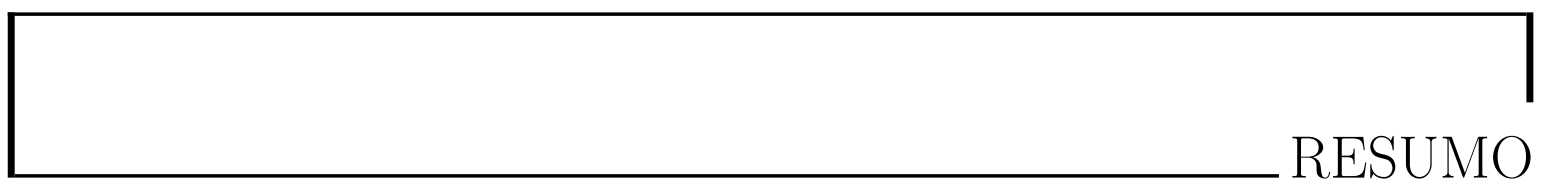

Seja K um conjunto de Cantor. Neste trabalho apresentamos dois teoremas relacionados a densidade do conjunto das dinâmicas simbólicas. No caso de endomorfismos provamos que, dado uma dinâmica $T: K \rightarrow K$, existe uma $\tilde{T}: K \rightarrow K$ próxima a $T$, tal que toda órbita é finalmente periódica. Já no caso de homeomorfismos, mostramos que, dado uma dinâmica $T: K \rightarrow K$, existe uma $\tilde{T}: K \rightarrow K$ próxima a $T$, tal que o w-limite de toda órbita de $\tilde{T}$ é uma órbita periódica. Em particular, mostramos que, em ambos os casos, todas as medidas ergódicas estão suportadas em órbitas periódicas.

Palavras-chave: conjunto de Cantor, endomorfismo, homeomorfismo, órbita finalmente periódica, órbita periódica. 



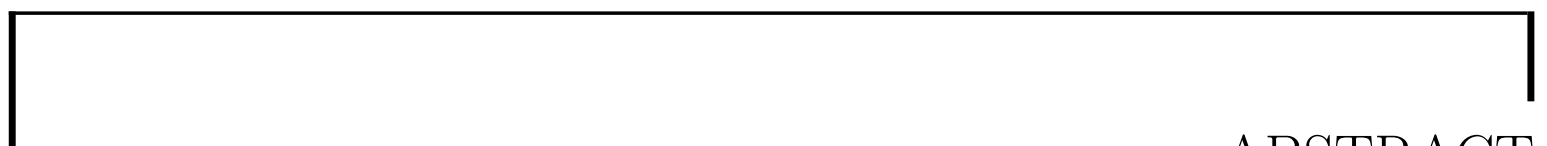
ABSTRACT

Let $K$ be a Cantor set. In this thesis we present two theorems related to the density of symbolic dynamics. We prove that given an endomorphism $T: K \rightarrow K$ then there exists an endomorphism $\tilde{T}: K \rightarrow K$ close to $T$ such that every orbit is finally periodic. We also prove that given a homeomorphism $T: K \rightarrow K$ then there exists a homeomorphism $\tilde{T}: K \rightarrow K$ close to $T$ such that the w-limit of every orbit is a periodic orbit. In particular, we have shown, in both cases, that all ergodic measures have support on periodic orbits.

Keywords: linear Cantor set, endomorphism, homeomorphism, finally periodic orbit, periodic orbit. 



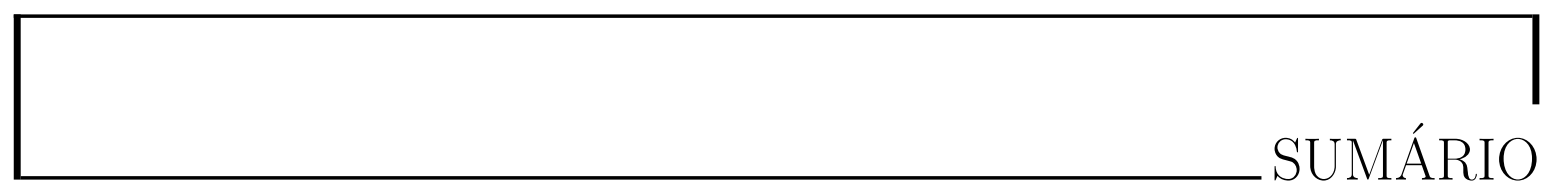

Introdução 2

1 Preliminares $\quad 6$

1.1 Teoria ergódica . . . . . . . . . . . . . . . . . 6

1.2 Otimização ergódica . . . . . . . . . . . . . . . . . . 14

1.3 Aproximações de dinâmicas . . . . . . . . . . . . . . . . . 23

2 Conjunto de Cantor $\quad 28$

2.1 O conjunto de Cantor . . . . . . . . . . . . . . . . . . . . 28

2.2 O conjunto ternário de Cantor . . . . . . . . . . . . . . . . 29

$2.3 \mathrm{O}$ conjunto das sequências . . . . . . . . . . . . . . . . 31

3 Primeiros resultados e exemplos 32

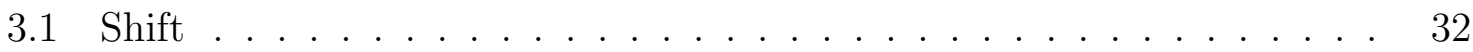

$3.2 \mathrm{O}$ espaço $\Sigma_{2}^{+} \ldots \ldots \ldots \ldots \ldots \ldots \ldots \ldots$

4 Resultado Principal $\quad 38$

4.1 Caso particular . . . . . . . . . . . . . . . . . 38

4.2 Resultados auxiliares . . . . . . . . . . . . . . . . . . . . 40

4.3 Endomorfismos . . . . . . . . . . . . . . . . . . . . 44 
4.4 Homeomorfismos . . . . . . . . . . . . . . . . . . . . . . . 47

$\begin{array}{ll}\text { Bibliografia } & 56\end{array}$ 
Sejam $X$ um espaço topológico, $T: X \rightarrow X$ e $f: X \rightarrow \mathbb{R}$ funções contínuas. Em otimização ergódica nos concentramos em maximizar o funcional $F_{f}: M_{T}(X) \rightarrow \mathbb{R}$ definido por:

$$
F_{f}(\mu)=\int_{X} f d \mu
$$

onde $M_{T}(X)$ é o conjunto das medidas de probabilidade borelianas $T$-invariantes.

Desde que $X$ seja compacto, essa medida maximizante existe e é o objeto de interesse nessa área. Consideremos o conjunto das dinâmicas simbólicas como o conjunto das dinâmicas definidas no espaço das sequências de N-símbolos.

Em uma das linhas de estudo da otimização ergódica, podemos fixar a função $f$ e analisar o que acontece com as medidas maximizantes para pertubações da dinâmica $T$. Nosso primeiro propósito nesse trabalho foi fazer essa análise no conjunto das dinâmicas simbólicas. Com isso, nos deparamos com um problema mais geral, e nosso trabalho passou a ser investigar a aproximação de dinâmicas simbólicas.

Um conjunto é dito um conjunto de Cantor se é totalmente desconexo, perfeito e compacto. Nessa tese, toda vez que nos referirmos a um conjunto de Cantor, estaremos tratando de um conjunto de Cantor num espaço métrico. Como, por um resultado clássico (veja [11]), para quaisquer dois conjuntos de cantor $K_{1}$ e $K_{2}$ sempre existe um homeomorfismo $h: K_{1} \rightarrow K_{2}$, e como todos os nossos resultados são topológicos, 
é indiferente a escolha do conjunto de Cantor que iremos trabalhar. Assim, sempre que necessário e para facilitar a compreensão dos resultados, poderemos considerar que o conjunto de Cantor que estamos trabalhando é o conjunto das sequências de $N$-símbolos com a métrica usual.

Os resultados principais estão divididos nos casos dos endomorfismos e dos homeomorfismos. Mais especificamente, demonstramos:

Teorema (Endomorfismos). Seja K um conjunto de Cantor. Dados um endomorfismo $T: K \rightarrow K$ e $\varepsilon>0$, existe um endomorfismo $\tilde{T}: K \rightarrow K$ tal que:

$$
D(T, \tilde{T})=\max _{x \in K} d(T(x), \tilde{T}(x))<\varepsilon
$$

e toda órbita de $\tilde{T}$ é finalmente periódica.

Teorema (Homeomorfismos). Seja K um conjunto de Cantor. Dados um homeomorfismo $T: K \rightarrow K$ e $\varepsilon>0$, existe um homeomorfismo $\tilde{T}: K \rightarrow K$ tal que:

$$
D(T, \tilde{T})=\max _{x \in K} d(T(x), \tilde{T}(x))<\varepsilon
$$

e o w-limite de toda órbita de T̃ é uma órbita periódica.

A partir desses teoremas, resolvemos o proposto dentro da otimização ergódica:

Corolário. Seja K um conjunto de Cantor. Dados um endomorfismo (respectivamente homeomorfismo) $T: K \rightarrow K$, uma função contínua $f: K \rightarrow \mathbb{R} e \varepsilon>0$, existe um endomorfismo (respectivamente homeomorfismo) $\tilde{T}: K \rightarrow K$ com

$$
D(T, \tilde{T})=\max _{x \in K} d(T(x), \tilde{T}(x))<\varepsilon
$$

e tal que existe uma medida f-maximizante suportada numa órbita periódica.

Nosso trabalho está organizado da seguinte maneira:

O capítulo 1 começa com as noções básicas da teoria ergódica. Em seguida, expõe as linhas de pesquisa e os principais resultados dentro da otimização ergódica, de onde 
segue a definição do problema, mais geral. No final desse capítulo, tratamos de algumas questões e respostas que aparecem com respeito a aproximação de dinâmicas.

O capítulo 2 introduz conceitos de dinâmicas simbólicas. Nele, colocamos as propriedades de um conjunto de Cantor e do conjunto das sequências, conceitos usados ao longo de nosso estudo.

O capítulo 3 apresenta o problema proposto para uma dinâmica fixa e coloca os primeiros exemplos que aparecem antes dos resultados finais.

O capítulo 4 é dedicado a demonstração dos dois teoremas principais. Para isso, trata também de casos particulares e de alguns resultados auxiliares sobre divisão de um conjunto de Cantor. 



\section{CAPÍTULO 1}

\section{$1.1 \quad$ Teoria ergódica}

Nesta seção lembraremos alguns conceitos da teoria ergódica que ajudam a entender o desenvolvimento desse trabalho. As referências para esse estudo são [18] e [29].

Definição 1.1.1. Sejam $(X, \mathcal{B}, \mu)$ um espaço de medida ${ }^{1}$ e $T: X \rightarrow X$ uma transformação mensurável. Dizemos que T preserva medida ou $\mu$ é T-invariante, se para todo $A \in \mathcal{B}$,

$$
\mu\left(T^{-1}(A)\right)=\mu(A) .
$$

Exemplo 1.1.2. Suponha que $X$ é um espaço topológico, $T: X \rightarrow X$ é contínua $e, p$ um ponto tal que $T^{n}(p)=p$. Seja $\delta_{x}$ a medida de Dirac associada a um ponto $x$ definida por:

\footnotetext{
${ }^{1}$ Em geral, $X$ é um espaço topológico, $\mathcal{B}$ é a $\sigma$-álgebra de Borel e $(X, \mathcal{B}, \mu)$ é de probabilidade.
} 


$$
\begin{aligned}
\delta_{x}(A)=\left\{\begin{array}{l}
1, \text { se } x \in A \\
0, \text { se } x \notin A
\end{array} .\right. \text { Então a medida } & \\
& \mu=\frac{1}{n} \sum_{j=0}^{n-1} \delta_{T^{j}(p)}
\end{aligned}
$$

é invariante por $T$.

De fato, para $A \subset X$ um conjunto mensurável qualquer, temos que $\mu\left(T^{-1}(A)\right)=\mu(A)$ :

$$
\begin{aligned}
\frac{1}{n} \sum_{j=0}^{n-1} \delta_{T^{j}(p)}\left(T^{-1}(A)\right) & =\frac{1}{n} \#\left\{0 \leq j \leq n-1, T^{j}(p) \in T^{-1}(A)\right\} \\
& =\frac{1}{n} \#\left\{0 \leq j \leq n-1, T^{j+1}(p) \in A\right\} \\
& =\frac{1}{n} \#\left\{1 \leq j \leq n, T^{j}(p) \in A\right\}
\end{aligned}
$$

Mas, $\operatorname{como} T^{n}(p)=p$,

$$
\begin{aligned}
\frac{1}{n} \#\left\{1 \leq j \leq n, T^{j}(p) \in A\right\} & =\frac{1}{n} \#\left\{0 \leq j \leq n-1, T^{j}(p) \in A\right\} \\
& =\frac{1}{n} \sum_{j=0}^{n-1} \delta_{T^{j}(p)}(A) .
\end{aligned}
$$

Lembramos que, para qualquer $f: X \rightarrow \mathbb{R}$,

$$
\int_{X} f d \delta_{x}=f(x)
$$

Considere $X$ um espaço métrico compacto e $\mathcal{M}(X)$ o conjunto de todas as medidas de probabilidade sobre os borelianos de $X$. Para uma transformação $T: X \rightarrow X$, definimos $\mathcal{M}_{T}(X) \subset \mathcal{M}(X)$ o conjunto das medidas invariantes por $T$.

A fim de definir uma métrica em $\mathcal{M}(X)$, considere $\mathcal{C}^{0}(X)$ o espaço vetorial das funções contínuas provido da norma do supremo $\|f\|_{\infty}=\sup _{x \in X}|f(x)|$. Como $X$ é métrico compacto, existe um conjunto enumerável $\left\{g_{i}\right\}_{i \in \mathbb{N}}$ denso na bola unitária

$$
B=\left\{f \in \mathcal{C}^{0}(X):\|f\|_{\infty} \leq 1\right\}
$$


Seja $\xi: \mathcal{C}^{0}(X) \rightarrow \mathbb{R}$ um funcional linear contínuo positivo, com $\xi(1)=1$. Pelo teorema da representação de Riesz, existe uma única medida de probabilidade $\mu \in$ $\mathcal{M}(X)$ tal que

$$
\int_{X} f d \mu=\xi(f)
$$

para toda $f \in \mathcal{C}^{0}(X)$. Assim, tomando o conjunto $\left\{g_{i}\right\}_{i \in \mathbb{N}}$, definimos em $\mathcal{M}(X)$ a seguinte métrica:

$$
d(\mu, v)=\sum_{i=1}^{\infty} \frac{1}{2^{i}}\left|\int_{X} g_{i} d \mu-\int_{X} g_{i} d v\right| .
$$

Teorema 1.1.3. $(\mathcal{M}(X), d)$ é um espaço métrico compacto.

Sobre o conjunto das medidas invariantes, podemos concluir:

Teorema 1.1.4. (Krylov-Bogolioubov) $\mathcal{M}_{T}(X) \neq \emptyset$.

\section{Demonstração.}

Consideremos a sequência $\left\{\mu_{n}\right\} \subset \mathcal{M}(X)$ dada por:

$$
\mu_{n}=\frac{1}{n} \sum_{k=0}^{n-1} \delta_{T^{k} x}
$$

Por causa da compacidade de $\mathcal{M}(X)$, existe subsequência $\left\{\mu_{n_{m}}\right\}$ que converge na topologia fraca-* a uma medida de probabilidade $\mu$, isto é,

$$
\int_{X} g d \mu=\lim _{m \rightarrow \infty} \int_{X} g d \mu_{n_{m}}
$$

para qualquer função contínua g.

Para verificar que $\mu$ é uma medida invariante, é suficiente mostrar que $\int_{X} g \circ T d \mu=\int_{X} g d \mu$ para toda função contínua $g$. Temos que $T$ é contínua então 
$g \circ T$ também é. Assim,

$$
\begin{aligned}
\int_{X} g \circ T d \mu & =\lim _{m \rightarrow \infty} \int_{X} g \circ T d \mu_{n_{m}} \\
& =\lim _{m \rightarrow \infty} \frac{1}{n_{m}} \sum_{k=0}^{n_{m}-1} g\left(T^{k+1}(x)\right) \\
& =\lim _{m \rightarrow \infty}\left(\frac{1}{n_{m}} \sum_{k=0}^{n_{m}-1} g\left(T^{k}(x)\right)+\frac{1}{n_{m}}\left[g\left(T^{n_{m}}(x)\right)-g(x)\right]\right) .
\end{aligned}
$$

Como $g$ é limitada em $X$,

$$
\int_{X} g \circ T d \mu=\lim _{m \rightarrow \infty} \frac{1}{n_{m}} \sum_{k=0}^{n_{m}-1} g\left(T^{k}(x)\right)=\lim _{m \rightarrow \infty} \int_{X} g d \mu_{n_{m}}=\int_{X} g d \mu .
$$

Logo, $\mathcal{M}_{T}(X) \neq \emptyset$.

Lema 1.1.5. $\mathcal{M}_{T}(X)$ é convexo e compacto.

\section{Demonstração.}

Mostremos que $\mathcal{M}_{T}(X)$ é convexo, ou seja, se para quaisquer $\mu_{1}, \mu_{2} \in \mathcal{M}_{T}(X)$ e para qualquer $t \in[0,1]$,

$$
\mu_{t}=(1-t) \mu_{1}+t \mu_{2} \in \mathcal{M}_{T}(X) .
$$

De fato,

$$
\mu_{t}(A)=(1-t) \mu_{1}(A)+t \mu_{2}(A)=(1-t) \mu_{1}\left(T^{-1} A\right)+t \mu_{2}\left(T^{-1} A\right)=\mu_{t}\left(T^{-1} A\right),
$$

para todo $A \subset X$ mensurável.

Mostremos que $\mathcal{M}_{T}(X)$ é fechado, ou seja, se $\mu_{n} \in \mathcal{M}_{T}(X)$ com $\mu_{n} \rightarrow \mu$, então $\mu \in \mathcal{M}_{T}(X)$.

De fato, considerando $f$ uma função contínua qualquer,

$$
\int_{X} f \circ T d \mu=\lim _{n \rightarrow \infty} \int_{X} f \circ T d \mu_{n}=\lim _{n \rightarrow \infty} \int_{X} f d \mu_{n}=\int_{X} f d \mu
$$


Logo, $\mu$ é uma medida invariante.

Além disso, temos que todo subconjunto fechado de um espaço topológico compacto é compacto. Como $\mathcal{M}(X)$ é compacto, então $\mathcal{M}_{T}(X) \subset \mathcal{M}(X)$ é compacto.

A fim de colocar o resultado fundamental da teoria ergódica, que diz respeito a média temporal, definimos:

Definição 1.1.6. Seja $(X, \mathcal{B}, \mu)$ um espaço de probabilidade. Uma transformação $T$ que preserva medida nesse espaço é chamada ergódica se todo conjunto invariante ${ }^{2}$ tem medida zero ou um.

Exemplo 1.1.7. A medida definida em 1.1.2 é ergódica, pois qualquer conjunto totalmente invariante ou contém ou não intersecta o conjunto $\left\{p, T(p), T^{2}(p), \ldots, T^{n-1}(p)\right\}$, que tem medida um.

Exemplo 1.1.8. Uma rotação do círculo é ergódica com respeito a medida de Lebesgue se, e somente se, for irracional.

Teorema 1.1.9. (ergódico de Birkhoff) Seja $(X, \mathcal{B}, \mu)$ um espaço de probabilidade e $T: X \rightarrow X$ uma transformação que preserva medida. Então,

1. se $f \in \mathcal{L}^{1}(X)$, o limite

$$
\tilde{f}(x):=\lim _{n \rightarrow \infty} \frac{1}{n} \sum_{j=0}^{n-1} f\left(T^{j}(x)\right)
$$

existe para quase todo ponto $x \in X$.

2. $\tilde{f}$ é uma função invariante, ou seja, $\tilde{f}(x)=\tilde{f}(T(x))$ quase todo ponto.

3. $\int_{X} \tilde{f} d \mu=\int_{X} f d \mu$.

${ }^{2} \mathrm{Um}$ conjunto $A \in \mathcal{B}$ é dito invariante por $T$ se $T^{-1}(A)=A$. 


$$
\text { 4. } \lim _{n \rightarrow \infty}\left\|\tilde{f}-\frac{1}{n} \sum_{j=0}^{n-1} f \circ T^{j}\right\|_{1}=0
$$

A seguir, veremos que a ergodicidade é condição suficiente e necessária para essa média temporal ser igual a média espacial em quase todo ponto. Mais precisamente, se $T$ é ergódica e $f \in \mathcal{L}^{1}(X)$,

$$
\lim _{n \rightarrow \infty} \frac{1}{n} \sum_{i=0}^{n-1} f\left(T^{i}(x)\right)=\int_{X} f d \mu,
$$

em $\mu$-quase todo ponto $x \in X$.

Lema 1.1.10. Seja T uma transformação que preserva medida em um espaço de probabilidade $(X, \mathcal{B}, \mu)$. T é ergódica se, e somente se, qualquer função mensurável $f: X \rightarrow \mathbb{R}$ invariante $\mu$-q.t.p. for constante $\mu$-q.t.p.

Demonstração. Primeiramente, suponha que toda função mensurável $f: X \rightarrow \mathbb{R}$ invariante $\mu$-q.t.p. é constante $\mu$-q.t.p.

Considere $B \in \mathcal{B}$ um conjunto invariante e tome $f=\chi_{B}$. Vejamos que $f$ é invariante:

$$
\begin{aligned}
\chi_{B}(x) & =\left\{\begin{array}{l}
1, \text { se } x \in B=T^{-1}(B) \\
0, \text { se } x \notin B=T^{-1}(B)
\end{array}\right. \\
& =\left\{\begin{array}{l}
1, \text { se } T(x) \in B \\
0, \text { se } T(x) \notin B
\end{array}\right. \\
& =\chi_{B}(T(x)) .
\end{aligned}
$$

Mas, para $\chi_{B}$ ser constante $\mu$-q.t.p., $\mu(B)=0$ ou $\mu(B)=1$. Portanto, $T$ é ergódica.

Suponha agora que existe $f: X \rightarrow \mathbb{R}$ mensurável, tal que $f=f \circ T$ quase todo ponto, e $f$ não é constante $\mu$-q.t.p. Logo, existe $M \in \mathbb{R}$ tal que, se

$$
E:=\{x \in X: f(x)<M\}
$$

então $0<\mu(E)<1$ e $0<\mu\left(E^{C}\right)<1$. 
Observe que $E$ é invariante, ou seja, $T(E)=E$. De fato, se $x \in E$ então $f(T(x))=f(x)<M$, implicando que $T(x) \in E$. Analogamente, se $T(x) \in E$ então $x \in E$.

Mas, $\mu(E)$ é diferente de zero e de um. Portanto, $\mu$ não é ergódica.

A respeito da caracterização das medidas de probabilidade ergódicas de $T$, temos que:

Lema 1.1.11. Seja $(X, \mathcal{B}, \mu)$ um espaço de medida e $T: X \rightarrow X$ contínua, onde $X$ é um espaço métrico compacto. Então $\mu$ é ergódica se, e somente se, $\mu$ é ponto extrema $l^{3}$ de $\mathcal{M}_{T}(X)$.

Se $\mathcal{M}_{T}(X)$ consistir de um único elemento, $T$ é dita unicamente ergódica.

Um outro conceito essencial é o suporte de uma medida:

Definição 1.1.12. Sejam $X$ espaço métrico separável e $\mu$ medida de probabilidade sobre os borelianos. O suporte de $\mu$ é dado por

$$
\operatorname{supp}(\mu)=\{x \in X: \forall \text { aberto } U \operatorname{com} x \in U, \mu(U)>0\}
$$

Lema 1.1.13. O suporte de $\mu$ é um conjunto fechado e $\mu(\operatorname{supp}(\mu))=1$.

O teorema descrito abaixo diz que cada medida de $\mathcal{M}_{T}(X)$ pode ser escrita em termos dos pontos extremais de $\mathcal{M}_{T}(X)$, ou seja, das medidas ergódicas.

Teorema 1.1.14. (decomposição ergódica) Sejam $X$ um espaço compacto e $T: X \rightarrow X$ uma função contínua. Então, para cada $\mu \in \mathcal{M}_{T}(X)$, existe $\rho_{\mu}$ uma

\footnotetext{
${ }^{3} \mu$ é ponto extremal de $\mathcal{M}_{T}(X)$ se, para quaisquer $\mu_{1}, \mu_{2} \in \mathcal{M}_{T}(X)$, com $\mu_{1} \neq \mu_{2}$, e $t \in[0,1]$, a identidade $\mu=t \mu_{1}+(1-t) \mu_{2}$ implicar que $t=0$ ou $t=1$.
} 
medida definida sobre os borelianos do espaço métrico compacto $\mathcal{M}_{T}(X)$, com a propriedade que $\rho_{\mu}\left(\operatorname{EM}_{T}(X)\right)=1$ e

$$
\mu=\int_{E \mathcal{M}_{T}(X)} m d \rho_{\mu}(m)
$$

onde $\operatorname{EM}_{T}(X)$ são as medidas ergódicas em $\mathcal{M}_{T}(X)$.

O próximo teorema merece destaque por suas várias aplicações:

Teorema 1.1.15. (recorrência de Poincaré) Seja $(X, \mathcal{B}, \mu)$ espaço de probabilidade e $T$ um automorfismo que preserva $\mu$ nesse espaço. Considere $E \in \mathcal{B}$ e $\hat{E} \in \mathcal{B}$ subconjunto de $E$ tal que seus pontos retornam infinitas vezes a $E$, isto é, $\hat{E}=E \cap\left(\bigcap_{n \geq 0} \bigcup_{i \geq n} T^{-i}(E)\right)$. Então $\mu(E)=\mu(\hat{E})$.

Demonstração. Considere $E_{n}=\bigcup_{i \geq n} T^{-i}(E)$, para $n \geq 0$.

Observe que $E_{0} \supset E_{1} \supset \ldots \supset E_{n} \supset \ldots$

Além disso, $T^{-1}\left(E_{i}\right)=E_{i+1}$, ou seja,

Segue que

$$
\mu\left(E_{i}\right)=\mu\left(T^{-1}\left(E_{i}\right)\right)=\mu\left(E_{i+1}\right) .
$$

$$
\mu\left(\bigcap_{n \geq 0} E_{n}\right)=\lim _{N \rightarrow \infty} \mu\left(E_{N}\right)=\lim _{N \rightarrow \infty} \mu\left(E_{0}\right)=\mu\left(E_{0}\right)
$$

Como $\bigcap_{n \geq 0} E_{n} \subset E_{0}$ e vale a igualdade acima, então existe $B \in \mathcal{B}$, com $\mu(B)=0$, tal que:

$$
\begin{gathered}
E_{0}=\bigcap_{n \geq 0} E_{n} \cup B \\
\Rightarrow \bigcap_{n \geq 0} E_{n}=E_{0} \cap B^{C},
\end{gathered}
$$

$\operatorname{com} \mu\left(B^{C}\right)=1$. Portanto,

$$
\mu(\hat{E})=\mu\left(E \cap \bigcap_{n \geq 0} E_{n}\right)=\mu\left(E \cap E_{0} \cap B^{C}\right) \stackrel{E \subseteq E_{0}}{=} \mu\left(E \cap B^{C}\right)=\mu(E) .
$$


A versão métrica desse resultado diz que, se $X$ é um espaço métrico separável e $\mathcal{B}$ a $\sigma$-álgebra de Borel, então $\mu$-quase todo ponto é recorrente, ou seja,

$$
\mu(\{p \in X: p \notin \omega(p)\})=0,
$$

onde $\omega(p)$ é chamado de $\omega$-limite ${ }^{4}$ de $p$.

\subsection{Otimização ergódica}

A teoria ergódica e a teoria de otimização são objetos de grande interesse na matemática. Nos últimos quinze anos, essas duas áreas se uniram na resolução de alguns problemas, consolidando uma nova linha de pesquisa. Para informações adicionais as colocadas aqui, recomendamos a leitura de [16].

Suponha uma aplicação $T: X \rightarrow X$ num espaço topológico $X$ e uma função $f: X \rightarrow \mathbb{R}$ contínua.

Em otimização ergódica estamos interessados em maximizar o funcional $P_{f}: M_{T}(X) \rightarrow \mathbb{R}$ definido por:

$$
P_{f}(\mu)=\int_{X} f d \mu
$$

onde $M_{T}(X)$ é o conjunto das medidas de probabilidade borelianas $T$-invariantes.

Associada a esse funcional, temos a média temporal:

$$
\lim _{n \rightarrow \infty} \frac{1}{n} S_{n} f(x)
$$

onde $S_{n} f=\sum_{i=0}^{n-1} f \circ T^{i}$.

Assim, estudar o valor máximo do funcional $P_{f}$ está associado a encontrar o supremo da média sobre todas as escolhas da posição inicial $x$. Mais do que isso, estamos particularmente interessados em verificar se para algum $x$, o supremo é atingido.

\footnotetext{
${ }^{4}$ Lembremos que $\omega(p):=\left\{x \in X: \exists\left\{n_{k}\right\}_{k \in \mathbb{N}} \subset \mathbb{N}\right.$ tal que $n_{k} \rightarrow \infty$ e $\left.\lim _{k \rightarrow \infty} T^{n_{k}}(x)=p\right\}$
} 
Em geral, o limite acima não precisa existir. Para contornar esse problema podemos usar dois caminhos. O primeiro é definirmos

$$
\operatorname{Reg}(X, T, f):=\left\{x \in X: \lim _{n \rightarrow \infty} \frac{1}{n} S_{n} f(x) \text { existe }\right\}
$$

e então calcularmos o supremo sobre esses pontos.

O segundo caminho é usar o lim sup no lugar do limite e, então, calcular o supremo sobre todos os pontos $x \in X$.

Considere:

$$
\mathcal{M}_{f}:=\left\{\mu \in \mathcal{M}_{T}: \int_{X} f d \mu \text { existe }\right\}
$$

A partir dessas motivações, podemos definir,

Definição 1.2.1. Seja $X$ um espaço topológico, e suponha que $f: X \rightarrow \mathbb{R}$ é contínua. Então:

$$
\begin{aligned}
& \alpha(f)=\alpha(f, T)=\sup _{m \in \mathcal{M}_{f}} \int_{X} f d m \\
& \beta(f)=\beta(f, T)=\sup _{x \in \operatorname{Reg}(X, T, f)} \lim _{n \rightarrow \infty} \frac{1}{n} S_{n} f(x) \\
& \gamma(f)=\gamma(f, T)=\sup _{x \in X} \limsup _{n \rightarrow \infty} \frac{1}{n} S_{n} f(x) \\
& \delta(f)=\delta(f, T)=\limsup _{n \rightarrow \infty} \frac{1}{n} \sup _{x \in X} S_{n} f(x) .
\end{aligned}
$$

Definição 1.2.2. Sejam $X$ um espaço topológico e uma aplicação $T: X \rightarrow X$. A quantidade

$$
\alpha(f)=\sup _{m \in \mathcal{M}_{f}} \int_{X} f d \mu
$$

é chamada de média ergódica máxima para $f$. Uma medida $\mu \in \mathcal{M}_{T}$ é chamada $f$-maximizante se $\alpha(f)=\int_{X} f d \mu$, e o conjunto de todas as medidas maximizantes é denotado por $\mathcal{M}_{\max }(f)$. 
Em geral, $\alpha(f), \beta(f), \gamma(f), \delta(f)$ não coincidem, embora possam ser impostas condições sobre $X$ e $T$ para que sejam iguais e então definir a média ergódica máxima.

Teorema 1.2.3. Seja $T: X \rightarrow X$ uma aplicação contínua num espaço métrico compacto. Se $f: X \rightarrow \mathbb{R}$ é contínua, ou uma função característica de um subconjunto fechado, então

$$
\alpha(f)=\beta(f)=\gamma(f)=\delta(f) \neq \pm \infty .
$$

Teorema 1.2.4. Se $X$ é um espaço métrico compacto e $f$ é contínua, então $\mathcal{M}_{\text {max }}(f) \neq \emptyset$.

\section{Demonstração.}

Considere $F: \mathcal{M}_{T}(X) \rightarrow(-\infty, \infty)$ definida por $F(\mu)=\int_{X} f d \mu$. Essa função é contínua na topologia fraca-*.

De fato, seja $\mu_{n} \rightarrow \mu$ em $\mathcal{M}_{T}(X)$. Então,

$$
\int_{X} g d \mu_{n} \rightarrow \int_{X} g d \mu
$$

para toda $g \in \mathcal{C}^{0}(X)$. Em particular,

$$
F\left(\mu_{n}\right) \rightarrow F(\mu)
$$

Além disso, como $X$ é um espaço métrico compacto, $\mathcal{M}_{T}(X)$ é compacto. Assim, segue que essa função assume máximo em $\mathcal{M}_{T}(X)$.

Com respeito a caracterização do conjunto das medidas maximizantes, temos o seguinte resultado:

Teorema 1.2.5. O conjunto $\left\{\mathcal{M}_{\max }(f): f \in \mathcal{C}(X)\right\}$ é precisamente o conjunto das faces ${ }^{5}$ fechadas de $\mathcal{M}_{T}$.

\footnotetext{
${ }^{5}$ Um subconjunto convexo e não-vazio $F K$ é chamado de face de $K$ se, quando $\lambda k_{1}+(1-\lambda) k_{2} \in F$ com $k_{1}, k_{2} \in K$ e $0<\lambda<1$, então $k_{1}, k_{2} \in F$.
} 
Dentro da otimização ergódica naturalmente surgem novas questões acerca do seu objeto de estudo, as medidas maximizantes. Colocamos aqui algumas respostas, mas note que como todas elas estão relacionadas, podemos dizer que são parciais.

No trabalho de O. Jenkinson [14] algumas condições são colocadas sobre $f$ para que as medidas maximizantes existam mesmo sem o espaço ser compacto, e mais do que isso, para que essas medidas possam ser caracterizadas em termos de seu suporte.

Definição 1.2.6. Dada uma função contínua $T: X \rightarrow X$, duas funções $f, g$ são chamadas cohomólogas se $f-g=\varphi-\varphi \circ T$, onde $\varphi$ é uma função contínua e limitada. Chamamos $\varphi$ de função cobordo.

Definição 1.2.7. Uma função contínua $\tilde{f} \sim f$ é chamada uma forma normal para $f$ se $\tilde{f}^{-1}(\sup \tilde{f}) \supset \operatorname{supp}(\mu)$, para alguma medida $\mu$ T-invariante.

Teorema 1.2.8. Suponha $T: X \rightarrow X$ uma função contínua no espaço topológico $X$. Assuma que a função contínua $f: X \rightarrow \mathbb{R}$ tem uma forma normal $\tilde{f}$. Então $\mathcal{M}_{f}=\mathcal{M}_{T}$ $e$

$$
\mathcal{M}_{\text {max }}(f)=\left\{\mu \in \mathcal{M}_{T}: \operatorname{supp}(\mu) \subset \tilde{f}^{-1}(\sup \tilde{f})\right\} \neq \emptyset
$$

A existência de uma forma normal pode ser garantida através do conceito de uma função ser essencialmente compacta, e é essa a idéia usada pelo autor.

Dando continuidade ao estudo em espaços não necessariamente compactos, em [15] particularmente temos o resultado para um subshift. Nesse trabalho, considerando um subshift unilateral, finitamente primitivo, do tipo finito sobre um alfabeto contável, é possível colocar condições sobre $f$ para que ela admita uma forma normal, ou seja, para que exista medida maximizante.

Já no trabalho de R. Bissacot e R. Freire [5], foi verificado que alguns desses casos podem ser relacionados com o caso compacto pois, com certas condições para $f$, existe medida maximizante cujo o suporte está contido num subshift compacto. Mais precisamente, considere $X_{A}(\mathbb{N})$ o subconjunto das sequências de $\mathbb{N}$ permissíveis pela matriz A. 
Definição 1.2.9. Dizemos que A é irredutível se, para qualquer $i, j \in \mathbb{N}$, existe uma palavra $w=w_{1} w_{2} \ldots, w_{k}$, tal que iwj é uma palavra permitida, ou seja, $A\left(i, w_{1}\right)=1$, $A\left(w_{i}, w_{i+1}\right)=1$ para $i=1,2, \ldots, k$, e $A\left(w_{k}, j\right)=1$.

Definição 1.2.10. Para uma função $f: X_{A}(\mathbb{N}) \rightarrow \mathbb{R}$, a j-ésima variação de $f$ é dada por:

$$
V_{j}(f)=\sup \left\{f(x)-f(y), \pi\left(\sigma^{i}(x)\right)=\pi\left(\sigma^{i}(y)\right) \text { para } i=0,1, \ldots, j-1\right\},
$$

onde $\pi: X_{A}(\mathbb{N}) \rightarrow \mathbb{N}$ é a projeção da primeira coordenada, ou seja, $\pi(x)=\pi\left(x_{0} x_{1} x_{2}, \ldots\right)=x_{0}$.

Dizemos que $f$ é de variação limitada se:

$$
V(f)=\sum_{j=1}^{\infty} V_{j}(f)<\infty .
$$

Definição 1.2.11. Uma função $f: X_{A}(\mathbb{N}) \rightarrow \mathbb{R}$ é dita coerciva se

$$
\left.\limsup _{i \rightarrow \infty} f\right|_{[i]}=-\infty
$$

onde $[i]:=\left\{x \in X_{A}(\mathbb{N}), \pi(x)=i\right\}$.

Teorema 1.2.12. Sejam $\sigma$ um shift sobre $X_{A}(\mathbb{N})$, com $A$ irredutivel, e $f: X_{A}(\mathbb{N}) \rightarrow \mathbb{R}$ uma função de variação limitada e coerciva. Então existe um subconjunto finito $\mathcal{A} \subset \mathbb{N}$ tal que $\left.A\right|_{\mathcal{A x \mathcal { A }}}$ é irredutivel $e$

$$
\sup _{\mu \in \mathcal{M}_{\sigma}\left(X_{A}(\mathbb{N})\right)} \int f d \mu=\sup _{\mu \in \mathcal{M}_{\sigma}\left(X_{A}(\mathcal{A})\right)} \int f d \mu .
$$

Mais do que isso,

$$
\operatorname{supp}\left(\mu_{\text {max }}\right) \subset \mathcal{M}_{\sigma}\left(X_{A}(\mathcal{A})\right)
$$

Considere agora a dinâmica $T$ fixa num espaço métrico compacto $X$.

Em [17], fixando também uma medida ergódica $\mu T$-invariante, temos a existência de uma função contínua $f$ tal que $\mu$ é a única medida que maximiza o funcional $P_{f}$ : 
Teorema 1.2.13. Seja $T: X \rightarrow X$ uma dinâmica contínua num espaço métrico compacto e suponha $\mu$ uma medida de probabilidade boreliana $T$-invariante sobre $X$. Então existe uma função contínua $f: X \rightarrow \mathbb{R}$ tal que $\mu$ é a única medida $f$-maximizante.

Ainda pensando numa dinâmica fixa, podemos pensar na caracterização do suporte das medidas maximizantes. No trabalho [16], considerando $X$ um espaço métrico compacto, provou-se que sobre certas condições colocadas em $T$, para uma função $f$ genérica no espaço das funções contínuas, existe uma única medida $f$-maximizante e, essa medida possui suporte total.

Nessa mesma linha, mas particularmente pensando na função que duplica o ângulo no círculo, em [6] temos:

Teorema 1.2.14. Considere $T: S^{1} \rightarrow S^{1}$ definida por $T(x)=2 x(\bmod 1)$. Para uma função $f \in C\left(S^{1}\right)$ genérica ${ }^{6}$, toda medida maximizante de $f$ tem suporte total.

Em [8], também considerando $T: S^{1} \rightarrow S^{1}$ e a dinâmica $T(x)=2 x(\bmod 1)$, para cada função $\rho_{w}(t)=\cos 2 \pi(t-w)$ temos que a medida $\rho$-maximizante é única. Além disso, ela está suportada numa órbita periódica para todo $w$, exceto para um conjunto de parâmetros $w$ de medida nula e dimensão de Hausdorff zero.

Uma outra maneira de abordar esses mesmos problemas é fazendo uma mudança na topologia.

Em [10], $X$ é um subshift unilateral do tipo finito. Para uma função continua $\alpha$-Holder genérica $f$, verificamos que existe uma única medida $f$-maximizante. Adicionalmente, no conjunto formado pela união das funções contínuas $\beta$-Holder, $\operatorname{com} \beta>\alpha \mathrm{e}$ com a topologia $C^{\alpha}$, genericamente a medida maximizante está suportada numa órbita periódica.

Em [6], T é fixada como o shift de Bernoulli e $\operatorname{Wal}(X, T)$ como o conjunto de todas as funções que satisfazem a condição de Walters. Então, obtemos que o con-

\footnotetext{
${ }^{6}$ Um conjunto de um espaço topológico é dito genérico se puder ser escrito com a interseção enumerável de conjuntos abertos densos.
} 
junto das funções em $\operatorname{Wal}(X, T)$ com uma medida maximizante periódica contém um subconjunto aberto e denso em $\operatorname{Wal}(X, T)$.

Uma outra direção a ser seguida nessa linha de pesquisa é encontrar a entropia das medidas maximizantes.

Para um espaço métrico compacto $X$ e uma dinâmica $T$ fixa, em [9] é demonstrado que a medida maximizante de uma função genérica em $C^{0}(X)$ tem entropia zero.

Em [20], assumindo que $X$ é um subshift do tipo finito, para uma função Holder contínua genérica, toda medida maximizante tem entropia zero.

Ainda relacionado a esses estudos, nosso trabalho é motivado por uma outra vertente. Podemos fixar a função $f$ e analisar o que acontece com as medidas maximizantes para pertubações da dinâmica $T$. Nestas condições, temos os seguintes resultados encontrados respectivamente em [26] e [27]:

Teorema 1.2.15. Seja End $\left(S^{1}\right)$ o conjunto dos endomorfismos do círculo. Então o conjunto das funções $T \in \operatorname{End}\left(S^{1}\right)$ tais que existe uma medida $f$-maximizante suportada em uma órbita periódica é denso no conjunto dos endomorfismos, com a topologia $C\left(S^{1}\right)$.

Teorema 1.2.16. Seja Hom $(X)$ o espaço dos homeomorfismos de uma variedade riemanniana compacta $X$. Então o conjunto das funções $T \in H o m(X)$ tais que existe uma medida $f$-maximizante suportada em uma órbita periódica é denso no conjunto dos homeomorfismos, com a topologia $C(X)$.

A generalização do primeiro teorema para uma variedade riemanniana compacta e conexa é feita em [12].

Em [28], vemos que não podemos ter genericidade:

Teorema 1.2.17. Sejam $X$ uma variedade riemanniana compacta e conexa de dimensão maior ou igual a dois, e $g: X \rightarrow \mathbb{R}$ uma função localmente não constante. Considere $\operatorname{Hom}_{\operatorname{Per}(T)}(X)$ o conjunto dos homeomorfismos $f: X \rightarrow X$ que 
tem medida f-maximizante suportada numa órbita periódica. Então o complementar de $\operatorname{Hom}_{P e r(T)}(X)$ é residual.

Diante dessas linhas de pesquisa, nosso primeiro propósito foi estudar um conjunto $X$ nas dinâmicas simbólicas em que, se $f: X \rightarrow \mathbb{R}$ é uma função contínua, então o conjunto das dinâmicas $T: X \rightarrow X$ tais que existe medida maximizante suportada em órbita periódica, é denso com a topologia $C(X)$.

Para isso, particularmente, podemos analisar a densidade do conjunto das dinâmicas simbólicas que tem todas suas órbitas periódicas. Ou seja, fomos conduzidos a resolver um problema mais geral, que não se refere somente a otimização ergódica.

Nossos resultados principais nesse trabalho (feitos no capítulo 4) são os dois seguintes teoremas:

Teorema (Endomorfismos). Seja K um conjunto de Cantor. Dados um endomorfismo $T: K \rightarrow K$ e $\varepsilon>0$, existe um endomorfismo $\tilde{T}: K \rightarrow K$ tal que:

$$
D(T, \tilde{T})=\max _{x \in K} d(T(x), \tilde{T}(x))<\varepsilon
$$

e toda órbita de $\tilde{T}$ é finalmente periódica ${ }^{7}$.

Teorema (Homeomorfismos). Seja K um conjunto de Cantor. Dados um homeomorfismo $T: K \rightarrow K$ e $\varepsilon>0$, existe um homeomorfismo $\tilde{T}: K \rightarrow K$ tal que:

$$
D(T, \tilde{T})=\max _{x \in K} d(T(x), \tilde{T}(x))<\varepsilon
$$

e o w-limite de toda órbita de $\tilde{T}$ é uma órbita periódica.

Como consequência desses teoremas, podemos utilizar:

Lema 1.2.18. Sejam $K$ um conjunto de Cantor e $\tilde{T}: K \rightarrow K$ uma dinâmica contínua. Se o w-limite de toda órbita de $\tilde{T}$ for uma órbita periódica então toda medida ergódica está suportada numa órbita periódica.

${ }^{7}$ Dizemos que a órbita de um ponto $x \in K$ é finalmente periódica pela $T$, se existem $j, N>0$ tais que $T^{N+j}(x)=T^{j}(x)$ 


\section{Demonstração.}

Denotemos por $P_{i}$ o conjunto dos pontos periódicos de período $i$ e, $P=\bigcup_{i \in \mathbb{N}} P_{i}$.

Considere $\mu$ uma medida ergódica. Mostremos que $\operatorname{supp}(\mu) \subset P_{N}$ para algum $N \geq 1$.

Observe que $\mu(P)=1$. De fato, o conjunto dos pontos recorrentes é igual ao conjunto $P$ pois, se $x \in K$ é um ponto recorrente de $\tilde{T}$ e $x$ converge para uma órbita periódica então $x$ é um ponto periódico.

Para cada $j \in \mathbb{N}$, defina $A_{j}=\bigcup_{i=1}^{j} P_{i}$.

Temos que, $1=\mu(P)=\lim _{j \rightarrow \infty} \mu\left(A_{j}\right)$ e, $\mu\left(P_{j}\right)=\mu\left(A_{j}\right)-\mu\left(A_{j-1}\right) \geq 0$, para qualquer $j \geq 2$. Como, para cada $j \in \mathbb{N}, A_{j}$ é um conjunto invariante, existe $N \geq 1$ tal que $\mu\left(P_{N}\right)=1$. E, como $P_{N}$ é fechado, segue que $\operatorname{supp}(\mu) \subset P_{N}$.

Mostremos que existe um conjunto unitário com medida positiva.

Sejam $A \subset K$ qualquer e

$$
B=\left(A \cap P_{N}\right) \bigcup \tilde{T}^{-1}\left(A \cap P_{N}\right) \bigcup \tilde{T}^{-2}\left(A \cap P_{N}\right) \bigcup \ldots \bigcup \tilde{T}^{-N+1}\left(A \cap P_{N}\right) .
$$

Como $B \subset \tilde{T}^{-1}(B)$ e $\mu$ é ergódica, então $\mu(B)=0$ ou $\mu(B)=1$.

Além disso, $\mu(B) \leq N \mu\left(A \cap P_{N}\right)=N \mu(A)$, ou seja, para qualquer $A \subset K, \mu(A)=0$ ou $\mu(A) \geq \frac{1}{N}$.

Assim, podemos tomar conjuntos $A_{i} \subset K$, com $i \in \mathbb{N}$, de maneira que $A_{1} \supset A_{2} \supset \ldots$, e satisfazendo que $\operatorname{diam}\left(A_{i}\right) \leq \frac{1}{2^{i}}$ e $\mu\left(A_{i}\right) \geq \frac{1}{N}$.

Logo, $\{p\}=\bigcap A_{i}$ é um conjunto unitário que satisfaz $\mu(\{p\}) \geq \frac{1}{N}$.

Como $\mu$ é uma medida ergódica que contém atômos ${ }^{8}$, então ela está suportada numa órbita periódica.

${ }^{8}$ Um conjunto $A \in \mathcal{B}$ é chamado um átomo se $\mu(A)>0$ e para todo $B \subset A$ temos que $\mu(B)=0$ ou $\mu(B)=\mu(A)$ 
Em particular, por 1.2.5, podemos tomar uma medida maximizante como uma medida ergódica. Assim, seguem os respectivos corolários que contribuem dentro da otimização ergódica:

Corolário. Seja K um conjunto de Cantor. Dados um endomorfismo (respectivamente homeomorfismo) $T: K \rightarrow K$, uma função contínua $f: K \rightarrow \mathbb{R}$ e $\varepsilon>0$, existe um endomorfismo (respectivamente homeomorfismo) $\tilde{T}: K \rightarrow K$ com

$$
D(T, \tilde{T})=\max _{x \in K} d(T(x), \tilde{T}(x))<\varepsilon
$$

e tal que existe uma medida f-maximizante suportada numa órbita periódica.

Já com os problemas definidos, podemos analisar alguns resultados existentes no que passa a ser o foco do nosso estudo, a aproximação de dinâmicas.

\subsection{Aproximações de dinâmicas}

Introduzir noções de aproximação para transformações que preservam medida é uma ferramenta que pode ser utilizada na investigação de problemas dentro da teoria ergódica.

Esse estudo se iniciou com P. Halmos, que utilizou a topologia de vizinhanças para conseguir os primeiros resultados nessa área.

Seja $A u t(X, \mathcal{B}, \mu)$ o grupo de todos os automorfismos borelianos que preservam medida num espaço de Borel padrão ${ }^{9}$. Um dos resultados obtidos em [13] é que o conjunto dos automorfismos ergódicos é genérico em $\operatorname{Aut}(X, \mathcal{B}, \mu)$.

Considere em $\operatorname{Aut}(X, \mathcal{B}, \mu)$ as métricas $d_{\mu}$ e $d_{w}$ definidas por:

$$
d_{\mu}(S, T)=\mu(\{x \in X: S(x) \neq T(x)\})
$$

para $S, T \in A u t(X, \mathcal{B}, \mu)$.

${ }^{9}(X, \mathcal{B})$ é um espaço de Borel padrão se é isomorfo a um espaço métrico completo com a $\sigma$-álgebra de Borel. 


$$
d_{w}(S, T)=\sum_{n=1}^{\infty} 2^{-n} \mu\left(S A_{n} \Delta T A_{n}\right)
$$

para $S, T \in \operatorname{Aut}(X, \mathcal{B}, \mu)$, e $\left(A_{n}\right)$ uma família contável de subconjuntos mensuráveis que geram $\mathcal{B}$.

A partir dessas métricas podemos considerar as respectivas topologias $\tau$ e $\tau_{w}$ geradas por elas, denominadas de uniforme e fraca. É com o uso dessas topologias que temos alguns resultados sobre a densidade de dinâmicas periódicas. Nesta direção, V. Rokhlin demonstra em [22], que o conjunto dos automorfismos periódicos é $d_{\mu}$-denso em $\operatorname{Aut}(X, \mathcal{B}, \mu)$.

É importante observarmos que as topologias definidas anteriormente podem ser definidas através de uma base de vizinhanças sobre $\operatorname{Aut}(X, \mathcal{B})$ :

Definição 1.3.1. As topologias $\tau$ e $\tau_{w}$ em Aut $(X, \mathcal{B})$ são definidas pelas bases de vizinhanças $\mathcal{U}=\left\{U\left(T ; \mu_{1}, \ldots, \mu_{n} ; \varepsilon\right)\right\}$ e $\mathcal{W}=\left\{W\left(T ; F_{1}, \ldots, F_{k} ; \varepsilon\right)\right\}$, respectivamente, onde

$$
\begin{aligned}
& U\left(T ; \mu_{1}, \mu_{2}, \ldots, \mu_{n} ; \varepsilon\right)=\left\{S \in \operatorname{Aut}(X, \mathcal{B}) \mid \mu_{i}(E(S, T))<\varepsilon, i=1,2, \ldots, n\right\}, \\
& W\left(T ; F_{1}, F_{2}, \ldots, F_{k} ; \varepsilon\right)=\left\{S \in \operatorname{Aut}(X, \mathcal{B}) \mid S F_{i}=T F_{i}<\varepsilon, i=1,2, \ldots, k\right\},
\end{aligned}
$$

$\operatorname{com} T \in \operatorname{Aut}(X, \mathcal{B}), \mu_{1}, \mu_{2}, \ldots, \mu_{n} \in \mathcal{M}(X), F_{1}, F_{2}, \ldots, F_{k} \in \mathcal{B}$ e $\varepsilon>0$.

Dando continuidade a esses estudos, S. Bezuglyi et. al. investigaram o grupo $A u t(X, \mathcal{B})$ introduzindo novas topologias e as relações existentes entre elas. Além de [2], num outro trabalho, os autores examinaram particularmente o conjunto dos homeomorfismos num conjunto de Cantor $\mathrm{C}$, denotado por Homeo $(C)$. Mais especificamente, em [3] temos:

Teorema 1.3.2. O conjunto Homeo $(C)$ é $\tau$-denso em Aut $(C, \mathcal{B})$.

Além disso, se $A_{p}$ é o conjunto de todos os homeomorfismos em Homeo $(C)$ não periódicos ${ }^{10}$, também temos:

\footnotetext{
${ }^{10} T \in H o m e o(C)$ é dito não periódico se $T$ não tem pontos periódicos
} 
Teorema 1.3.3. $A_{p}$ é denso em $\operatorname{Homeo}(C)$ com respeito a topologia $\tau_{w}$.

Destacamos que a topologia $\tau$ é equivalente a topologia gerada pela métrica $D$ :

$$
D(S, T)=\sup _{x \in C} d(S(x), T(x))
$$

para $S, T \in H o m e o(C)$. Já a topologia $\tau_{w}$ não é comparável com essa topologia, mais fina. A métrica $D$ será a utilizada no decorrer do nosso trabalho.

Analisando as propriedades topológicas do grupo Homeo $(C)$, através do conceito de topologicamente livre, K. Medynets, em [19], extendeu a classe de homeomorfismos não periódicos para conseguir a densidade com relação a $\tau$.

Ainda explorando os resultados existentes sobre densidade de homeomorfismos, considere o conjunto de todos os homeomorfismos expansivos ${ }^{11}$ de $C$. Em [23], M. Sears mostra que esse conjunto é denso em $\mathrm{Homeo}(C)$.

Dando continuidade a esse trabalho, podemos definir a propriedade de pseudoórbitas para homeomorfismos num espaço métrico $(X, d)$ qualquer:

Definição 1.3.4. Uma sequência $\left(x_{i}\right)_{i=-\infty}^{i=\infty}$ é dita ser uma $\delta$-pseudo órbita se $d\left(x_{i}, x_{i+1}\right)<\delta$ para todo $i \in \mathbb{Z}$.

$(X, T)$ é dito com a propriedade de sombreamento de pseudo-órbita se, dado $\varepsilon>0$, existe $\delta>0$ tal que, para toda $\delta$-pseudo órbita $\left(x_{i}\right)_{i=-\infty}^{i=\infty} \subset X$, existe $x \in X$ com $d\left(T^{i}(x), x_{i}\right)<\varepsilon$ para $i \in \mathbb{Z}$.

T. Shimomura [24] mostra que o conjunto dos homeomorfismos expansivos de $C$ com a propriedade de pseudo-órbitas é denso em Homeo $(C)$. Mais do que isso, um homeomorfismo de $C$ topologicamente transitivo (respectivamente mixing) é aproximado uniformemente por homeomorfismos expansivos topologicamente transitivos (respectivamente mixing) com a propriedade de pseudo-órbita. A topologia tratada para esse

\footnotetext{
${ }^{11}$ Um homeomorfismo em um conjunto de Cantor é expansivo se, e somente se, é topologicamente conjugado a um subshift
} 
resultado é a da convergência uniforme.

Observamos que, depois da escrita dessa tese, chegou ao nosso conhecimento o artigo de N. Bernardes Jr. e U. Darji [1], cujo os resultados facilitariam no desenvolvimento do nosso trabalho. 



\section{CAPÍTULO 2}

Antes de investigarmos as dinâmicas simbólicas, estudaremos as propriedades dos conjuntos que serão abordados.

\subsection{O conjunto de Cantor}

A fim de definir e estudar os conjuntos de Cantor, lembraremos alguns conceitos topológicos. Para isso, considere $X$ um espaço topológico e $S \subset X$ um subconjunto.

Definição 2.1.1. S é totalmente desconexo se cada compenente conexa é um ponto.

Definição 2.1.2. S é perfeito se é fechado e cada um de seus pontos é um ponto de acumulação de $S$.

Definição 2.1.3. S é denso em lugar nenhum se o interior do seu fecho é vazio, ou seja, $\operatorname{int}(\bar{S})=\emptyset$.

Assim, podemos formalizar o conceito essencial em nosso trabalho: 
Definição 2.1.4. Seja $X$ um espaço métrico e $K \subset X$ um subconjunto. $K$ é dito um conjunto de Cantor se é totalmente desconexo, perfeito e compacto.

Um resultado que será muito utilizado no decorrer desse trabalho pode ser encontrado em [11]:

Teorema 2.1.5. Num espaço métrico, dois subconjuntos perfeitos, compactos e totalmente desconexos, são homeomorfos.

\subsection{O conjunto ternário de Cantor}

A seguir, construiremos na reta um exemplo em que é possivel verificar as características de um conjunto de Cantor.

Exemplo 2.2.1. Sejam $I_{0}=[0,1]$

$$
\begin{aligned}
I_{1} & =\left[0, \frac{1}{3}\right] \cup\left[\frac{2}{3}, 1\right] \\
I_{2} & =\left[0, \frac{1}{9}\right] \cup\left[\frac{2}{9}, \frac{3}{9}\right] \cup\left[\frac{6}{9}, \frac{7}{9}\right] \cup\left[\frac{8}{9}, 1\right] \\
& =\left[0, \frac{1}{9}\right] \cup\left[\frac{2}{9}, \frac{1}{3}\right] \cup\left[\frac{2}{3}, \frac{7}{9}\right] \cup\left[\frac{8}{9}, 1\right] \\
\vdots & \\
I_{n} & =I_{n-1}-\bigcup_{k=0}^{\infty}\left(\frac{1+3 k}{3^{n}}, \frac{2+3 k}{3^{n}}\right), n \geq 1 .
\end{aligned}
$$

Observe que para cada $n \in \mathbb{N}, I_{n}$ é a união de $2^{n}$ intervalos fechados $I(n, j)$, ou seja, $I_{n}=\bigcup_{j=1}^{2^{n}} I(n, j)$.

Temos também que, para cada $n \in \mathbb{N}, I(n, j)$ tem comprimento $\frac{1}{3^{n}}$, com $1 \leq j \leq 2^{n}$. 

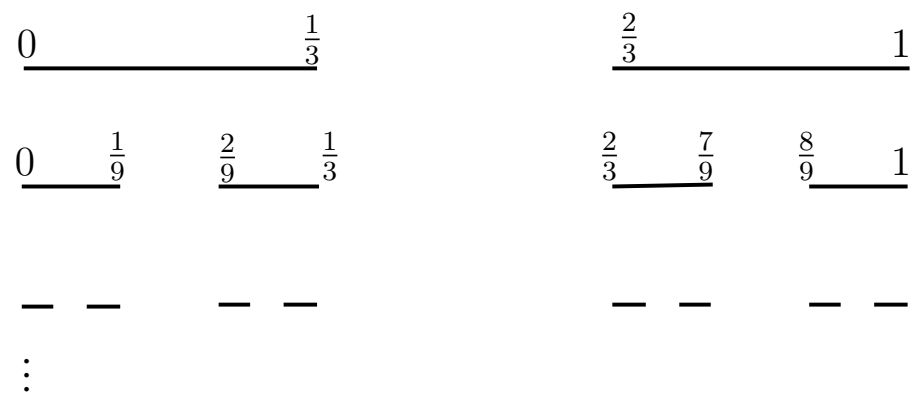

A interseção $C=\bigcap_{n=1}^{\infty} I_{n}$ é um conjunto de Cantor.

\section{Demonstração.}

i) $C$ é compacto, pois é a interseção não vazia de uma família de fechados limitados na reta.

ii) Mostremos que $C$ é perfeito.

Sejam $x \in C$ e $\varepsilon>0$ quaisquer. Tome $n \in \mathbb{N}$ tal que $\frac{1}{3^{n}}<\varepsilon$. Então $x \in I_{n}$, ou seja, $x \in I(n, j)$ para algum $1 \leq j \leq 2^{n}$.

Observe que, $I \subset(x-\varepsilon, x+\varepsilon)$, pois cada intervalo $I(n, j)$ tem comprimento $\frac{1}{3^{n}}<\varepsilon$.

Considere $y \neq x$ uma das extremidades de $I(n, j)$. Assim, como $y \in C$ e $0<|x-y|<\varepsilon, x$ é um ponto de acumulação de $C$.

iii) Mostremos que $C$ é totalmente desconexo.

Na reta, um subconjunto é totalmente desconexo se não contém intervalos. Assim, mostremos que $C$ não contém nenhum intervalo $] a, b[$.

Para qualquer $n \in \mathbb{N}$ temos que $C \subset \bigcup_{j=0}^{2^{n}} I(n, j)$, ou seja, $C$ está contido numa união disjunta .

Tome $n$ tal que $\frac{1}{2^{n}}<b-a$, pois assim cada $I_{n}$ tem comprimento inferior a $b-a$.

Portanto, $] a, b\left[\not \subset \bigcup_{j=1}^{2^{n}} I(n, j)\right.$.

Segue de i) ii) e iii) que $C$ é um conjunto de Cantor. 
Propriedade 2.2.2. Todo ponto $x \in C$ tem uma única expansão ternária: $x=\sum_{n=1}^{\infty} \frac{a_{n}}{3^{n}}$, onde cada $a_{n}$ é 0 ou 2 .

\section{$2.3 \mathrm{O}$ conjunto das sequências}

Seja $X$ o espaço das sequências $\left(x_{n}\right)_{n \in M}$, onde $M=\mathbb{Z}$ ou $M=\mathbb{N}$, e $x_{n} \in\{0,2\}$.

Se $M=\mathbb{Z}$ denotamos esse espaço por $\Sigma_{2}$ e, para quaisquer $x=\left(\ldots, x_{-2}, x_{-1}, x_{0}, x_{1}, x_{2}, \ldots\right) \in \Sigma_{2}$ e $y=\left(\ldots, y_{-2}, y_{-1}, y_{0}, y_{1}, y_{2}, \ldots\right) \in \Sigma_{2}$, definimos a métrica $d^{\prime}$ dada por:

$$
d^{\prime}(x, y)=\sum_{i \in M} \frac{\left|x_{i}-y_{i}\right|}{4^{|i|}}
$$

Se $M=\mathbb{N}$ denotamos esse espaço por $\Sigma_{2}^{+}$e, para quaisquer $x=\left(x_{1}, x_{2}, x_{3}, \ldots\right) \in \Sigma_{2}^{+}$ e $y=\left(y_{1}, y_{2}, y_{3}, \ldots\right) \in \Sigma_{2}^{+}$, definimos a métrica $d$ por:

$$
d(x, y)=\sum_{i \in M} \frac{\left|x_{i}-y_{i}\right|}{4^{i-1}}
$$

Podemos também considerar o espaço $\Sigma_{N}^{+}$, em que $M=\mathbb{N}$ e $x_{n} \in\{1,2,3, \ldots, N\}$. Para quaisquer $x=\left(x_{1}, x_{2}, x_{3}, \ldots\right) \in \Sigma_{N}^{+}$e $y=\left(y_{1}, y_{2}, y_{3}, \ldots\right) \in \Sigma_{N}^{+}$, utilizamos a métrica $\bar{d}$ por:

$$
\bar{d}(x, y)=2^{-\min \left\{i: x_{i} \neq y_{i}\right\}}
$$

cuja a qual é equivalente a $d$ para o caso $N=2$.

Proposição 2.3.1. O espaço das sequências $\Sigma_{2}^{+}$é um conjunto de Cantor.

\section{Demonstração.}

Sabemos que dois conjuntos de Cantor são homeomorfos (2.1.5). Assim, basta considerar a expansão ternária e definir o homeomorfismo $f: C \rightarrow \Sigma_{2}^{+}$dado por:

$$
\sum_{n=1}^{\infty} \frac{a_{n}}{3^{n}} \longmapsto\left(a_{1}, a_{2}, \ldots, a_{n}, \ldots\right) .
$$




\section{CAPÍTULO 3}

PRIMEIROS RESULTADOS E EXEMPLOS

Iniciamos nosso trabalho explorando alguns conjuntos $X$ e algumas dinâmicas $T: X \rightarrow X$ em que existe $\tilde{T}: X \rightarrow X$ próxima de $T$ tal que toda órbita é periódica.

\subsection{Shift}

Considere o homemomorfismo, conhecido como shift bilateral, definido por:

$$
\begin{aligned}
& \Sigma_{2} \rightarrow \Sigma_{2} \\
& \sigma:\left(x_{k}\right)_{k=-\infty}^{\infty}=\left(x_{k+1}\right)_{k=-\infty}^{\infty}
\end{aligned}
$$

Teorema 3.1.1. Dados $\varepsilon>0$ e o shift bilateral $\sigma$, existe um homeomorfismo $T: \Sigma_{2} \rightarrow \Sigma_{2}$ periódico tal que $D(T, \sigma)=\max _{x \in \Sigma_{2}} d^{\prime}(T(x), \sigma(x))<\varepsilon$.

Demonstração. Dado $\varepsilon>0$, existe $N>0$ suficientemente grande tal que $\frac{1}{N}<\varepsilon$.

Tome $n>0$ de modo que $\sum_{i=n}^{\infty} \frac{1}{2^{i}}<\frac{1}{N}$.

Definimos $T: \Sigma_{2} \rightarrow \Sigma_{2}$ por: $^{1}$

\footnotetext{
${ }^{1}$ Para melhor entendimento, indicaremos o elemento da posição 0 com uma barra.
} 


$$
\begin{aligned}
& T(\ldots, x_{-n-2}, x_{-n-1}, \underbrace{x_{-n}, x_{-n+1}, \ldots, x_{-1}, \overline{x_{0}}, x_{1}, \ldots, x_{n-1}, x_{n}}, x_{n+1}, x_{n+2}, \ldots)= \\
& (\ldots, x_{n+3}, x_{n+2}, \underbrace{x_{-n+1}, x_{-n+2}, \ldots, x_{0}, \overline{x_{1}}, x_{2}, \ldots, x_{n}, x_{n+1}}, x_{-n}, x_{-n-1}, x_{-n-2}, \ldots)
\end{aligned}
$$

i) Mostremos que $T$ é um homeomorfismo.

- $T$ é contínua: Dado $\bar{\varepsilon}>0$, existe $\bar{N}>0$ suficientemente grande tal que $\frac{1}{\bar{N}}<\bar{\varepsilon}$.

Seja $\bar{n}$ tal que $\sum_{i=\bar{n}}^{\infty} \frac{1}{2^{i}}<\frac{1}{\bar{N}}$. Tome $0<\delta<\sum_{i=\bar{n}+1}^{\infty} \frac{1}{2^{i}}$.

Assim, temos que para quaisquer $x, y \in \Sigma_{2}$, se $d^{\prime}(x, y)<\delta$, então $x_{i}=y_{i}$ para $-\bar{n}-1 \leq i \leq \bar{n}+1$, e portanto $T(x)$ e $T(y)$ coincidem nas $\bar{n}$-ésimas coordenadas centrais, implicando que:

$$
d^{\prime}(T(x), T(y))<\sum_{i=\bar{n}}^{\infty} \frac{1}{2^{i}}<\frac{1}{\bar{N}}<\bar{\varepsilon}
$$

- $T$ é sobrejetora: Dado $y=\left(\ldots, x_{-n}, x_{-n+1}, \ldots, x_{-1}, \overline{x_{0}}, x_{1}, \ldots, x_{n}, \ldots\right)$, existe

$$
x=(\ldots, x_{n+2}, \underbrace{x_{n+1}, x_{-n}, x_{-n+1}, \ldots, \overline{x_{-1}}, x_{0}, x_{1}, \ldots, x_{n-1}}, x_{n}, x_{-n-1}, x_{-n-2}, \ldots) \text { tal }
$$

que $T(x)=y$.

- $T$ é injetora: Dados $T(x)=T(y)$,

$$
\begin{aligned}
& \left(\ldots, x_{n+2}, x_{-n+1}, x_{-n+2}, \ldots, x_{0}, \overline{x_{1}}, x_{2}, \ldots, x_{n}, x_{n+1}, x_{-n}, x_{-n-1}, x_{-n-2}, \ldots\right)= \\
= & \left(\ldots, y_{n+2}, y_{-n+1}, y_{-n+2}, \ldots, y_{0}, \overline{y_{1}}, y_{2}, \ldots, y_{n}, y_{n+1}, y_{-n}, y_{-n-1}, y_{-n-2}, \ldots\right) .
\end{aligned}
$$

Segue que $x_{i}=y_{i}$ para todo $i \in \mathbb{Z}$, ou seja, $x=y$.

ii) Mostremos que $D(T, \sigma)<\varepsilon$.

$$
\begin{aligned}
D(\sigma, T) & =\max _{x \in \Sigma_{2}} d^{\prime}(\sigma(x), T(x)) \\
& =\max _{x \in \Sigma_{2}}\left[\sum_{i=-\infty}^{-n+1} \frac{\left|x_{i}-y_{i}\right|}{4^{-i}}+\sum_{i=n+1}^{\infty} \frac{\left|x_{i}-y_{i}\right|}{4^{i}}\right] \\
& \leq \sum_{i=n+1}^{\infty} 2 \frac{1}{4^{i}}=\sum_{i=n+1}^{\infty} \frac{1}{2^{i}}<\frac{1}{N}<\varepsilon
\end{aligned}
$$


iii) Verifiquemos que $T^{2 n+2}(x)=T(x)$.

Para isso, consideremos $n$ par. Analogamente vale para $n$ ímpar:

$$
\begin{aligned}
& x=(\ldots, x_{-n-2}, x_{-n-1}, \underbrace{x_{-n}, x_{-n+1}, \ldots, x_{-1}, \overline{x_{0}}, x_{1}, \ldots, x_{n-1}, x_{n}}, x_{n+1}, x_{n+2}, \ldots) \\
& T(x)=(\ldots, x_{n+3}, x_{n+2}, \underbrace{x_{-n+1}, x_{-n+2}, \ldots, x_{0}, \overline{x_{1}}, x_{2}, \ldots, x_{n}, x_{n+1}}, x_{-n}, x_{-n-1}, \ldots) \\
& T^{2}(x)=(\ldots, x_{-n-2}, x_{-n-1}, \underbrace{x_{-n+2}, x_{-n+3}, \ldots, x_{1}, \overline{x_{2}}, x_{3}, \ldots, x_{n+1}, x_{-n}}, x_{-n+1}, x_{n+2}, \ldots) \\
& T^{3}(x)=(\ldots, x_{n+3}, x_{n+2}, \underbrace{x_{-n+3}, x_{-n+4}, \ldots, x_{2}, \overline{x_{3}}, x_{4}, \ldots, x_{-n}, x_{-n+1}}, x_{-n+2}, x_{-n-1}, \ldots) \\
& \vdots \\
& T^{n}(x)=(\ldots, x_{-n-2}, x_{-n-1}, \underbrace{x_{0}, x_{1}, \ldots, x_{n-1}, \overline{x_{n}}, x_{n+1}, x_{-n}, \ldots, x_{-3}, x_{-2}}, x_{-1}, x_{n+2}, \ldots) \\
& T^{n+1}(x)=(\ldots, x_{n+3}, x_{n+2}, \underbrace{x_{1}, x_{2}, \ldots, x_{n}, \overline{x_{n+1}}, x_{-n}, \ldots, x_{-2}, x_{-1}}, x_{0}, x_{-n-1}, \ldots) \\
& T^{n+2}(x)=(\ldots, x_{-n-2}, x_{-n-1}, \underbrace{x_{2}, x_{3}, \ldots, x_{n+1}, \overline{x_{-n}}, x_{-n+1}, \ldots, x_{-1}, x_{0}}, x_{1}, x_{n+2}, \ldots) \\
& T^{n+3}(x)=(\ldots, x_{n+3}, x_{n+2}, \underbrace{x_{3}, x_{4}, \ldots, x_{n+1}, x_{-n}, \overline{x_{-n+1}}, x_{-n+2}, \ldots, x_{0}, x_{1}}, x_{2}, x_{-n-1}, \ldots) \\
& T^{2 n}(x)=(\ldots, x_{-n-2}, x_{-n-1}, \underbrace{x_{n}, x_{n+1}, x_{-n}, \ldots, x_{-3}, \overline{x_{-2}}}, x_{-1}, \ldots, x_{-3}, x_{-2}, x_{n-1}, x_{n+2}, \ldots) \\
& T^{2 n+1}(x)=(\ldots, x_{-n-2}, x_{-n-1}, \underbrace{x_{n+1}, x_{-n}, \ldots, x_{-2}, \overline{x_{-1}}, x_{0}, \ldots, x_{-2}, x_{-1}}, x_{n}, x_{n+2}, \ldots) \\
& T^{2 n+2}(x)=(\ldots, x_{-n-2}, x_{-n-1}, \underbrace{x_{-n}, \ldots, x_{-1}, \bar{x}_{0}, x_{1}, \ldots, x_{n-1}, x_{n}}, x_{n+1}, x_{n+2}, \ldots)=x
\end{aligned}
$$

Exemplo 3.1.2. No teorema anterior, se $\varepsilon>0$ é tal que podemos tomar $n=2$, temos:

$$
\begin{aligned}
x= & (\ldots, 0,0, \underbrace{0,0, \overline{2}, 0,0,2,2}, 2, \ldots) \\
T(x) & =(\ldots, 2,2,2, \underbrace{0,2, \overline{0}, 0,2}, 0,0,0, \ldots) \\
T^{2}(x) & =(\ldots, 0,0,0, \underbrace{2,0, \overline{0}, 2,0}, 0,2,2, \ldots) \\
T^{3}(x) & =(\ldots, 2,2,2, \underbrace{0,0, \overline{2}, 0,0}, 2,0,0, \ldots) \\
T^{4}(x) & =(\ldots, 0,0,0, \underbrace{0,2, \overline{0}, 0,2}, 0,2,2, \ldots) \\
T^{5}(x) & =(\ldots, 2,2,2, \underbrace{2,0, \overline{0}, 2,0}, 0,0,0, \ldots) \\
T^{6}(x) & =(\ldots, 0,0,0, \underbrace{0,0, \overline{2}, 0,0}, 2,2,2, \ldots)=x
\end{aligned}
$$

Através do próximo exemplo destacamos que, se $T$ não é o shift, nem sempre é possivel encontrar uma dinâmica próxima de $T$ com todas as suas óbitas periódicas. 
Exemplo 3.1.3. Considere $T:\{0,2\}^{\mathbb{N}} \rightarrow\{0,2\}^{\mathbb{N}}$ definida por:

$$
T(x)=\left\{\begin{array}{l}
\left(2, x_{3}, x_{4}, \ldots\right) \text { se } x=\left(2, x_{2}, x_{3}, x_{4}, \ldots\right) \\
\left(x_{2}, x_{3}, x_{4}, \ldots\right) \text { se } x=\left(0, x_{2}, x_{3}, x_{4}, \ldots\right)
\end{array}\right.
$$

Não existe $\tilde{T}$ próxima de $T$ tal que toda órbita seja periódica. Basta olhar para os pontos da forma $x=\left(0,1, x_{3}, x_{4}, \ldots\right)$.

Com isso, nosso trabalho passa a procurar dinâmicas finalmente periódicas.

Para simplificar nosso problema, consideramos nos primeiros casos uma distância pequena entre endomorfismos $T$ e $\tilde{T}$.

\section{$3.2 \mathrm{O}$ espaço $\Sigma_{2}^{+}$}

Nessa seção definiremos endomorfismos no espaço das sequências $\Sigma_{2}^{+}$, finalmente periódicos que estejam $\frac{1}{2}$-próximos de um endomorfismo $T: \Sigma_{2}^{+} \rightarrow \Sigma_{2}^{+}$qualquer. A generalização e os detalhes serão feitos no capitulo posterior.

Considere:

$$
\begin{aligned}
& W_{0}=\left\{x: x=\left(0, x_{2}, x_{3}, \ldots\right) \in \Sigma_{2}^{+}\right\} \\
& W_{2}=\left\{x: x=\left(2, x_{2}, x_{3}, \ldots\right) \in \Sigma_{2}^{+}\right\}
\end{aligned}
$$

Estudaremos os casos a partir da definição de $T$.

1. Existe $x \in W_{0}$ tal que $T(x) \in W_{0}$

Como $T$ é contínua, existe uma palavra $R_{0}$ de tamanho $l_{0}$ tal que $T\left(0, R_{0}, x_{l_{0}+2}, x_{l_{0}+3}, \ldots\right) \in W_{0}$.

Seja $W_{0,0}=\left\{x: x=\left(0, R_{0}, x_{l_{0}+2}, x_{l_{0}+3}, \ldots\right) \in \Sigma_{2}^{+}\right\}$.

(a) Existe $x \in W_{2}$ tal que $T(x) \in W_{2}$

Como $T$ é contínua, existe uma palavra $R_{2}$ de tamanho $l_{2}$ tal que $T\left(2, R_{2}, x_{l_{2}+2}, x_{l_{2}+3}, \ldots\right) \in W_{2}$.

Seja $W_{2,2}=\left\{x: x=\left(2, R_{2}, x_{l_{2}+2}, x_{l_{2}+3}, \ldots\right) \in \Sigma_{2}^{+}\right\}$. 

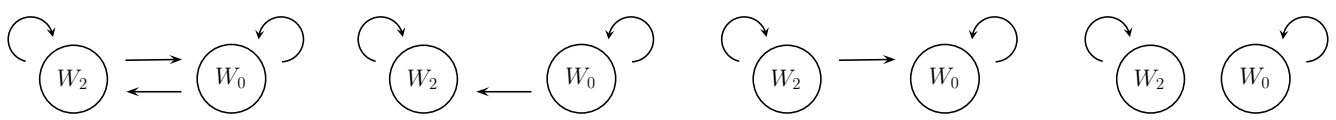

Definimos: $\tilde{T}(x)=\left\{\begin{array}{l}\left(0, R_{0}, R_{0}, R_{0}, \ldots\right) \text { se } T(x) \in W_{0} \text { mas } x \notin W_{0,0} \\ \left(0, x_{l_{0}+2}, \ldots\right) \text { se } x \in W_{0,0} \\ \left(2, R_{2}, R_{2}, R_{2}, \ldots\right) \text { se } T(x) \in W_{2} \text { mas } x \notin W_{2,2} \\ \left(2, x_{l_{2}+2}, \ldots\right) \text { se } x \in W_{2,2}\end{array}\right.$

(b) Para qualquer $x \in W_{2}, T(x) \in W_{0}$

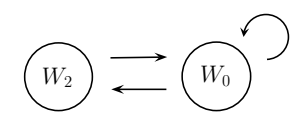

Definimos: $\tilde{T}(x)=\left\{\begin{array}{l}\left(0, R_{0}, R_{0}, R_{0}, \ldots\right) \text { se } T(x) \in W_{0} \text { mas } x \notin W_{0,0} \\ \left(0, x_{l_{0}+2}, \ldots\right) \text { se } x \in W_{0,0} \\ T(x) \text { se } T(x) \in W_{2}\end{array}\right.$

2. Para qualquer $x \in W_{0}, T(x) \in W_{2}$

(a) Existe $x \in W_{2}$ tal que $T(x) \in W_{2}$

Como $T$ é contínua, existe uma palavra $R_{2}$ de tamanho $l_{2}$ tal que $T\left(2, R_{2}, x_{l_{2}+2}, x_{l_{2}+3}, \ldots\right) \in W_{2}$.

Seja $W_{2,2}=\left\{x: x=\left(2, R_{2}, x_{l_{2}+2}, x_{l_{2}+3}, \ldots\right) \in \Sigma_{2}^{+}\right\}$.

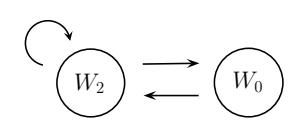

Definimos: $\tilde{T}(x)=\left\{\begin{array}{l}\left(2, R_{2}, R_{2}, R_{2}, \ldots\right) \text { se } T(x) \in W_{2} \text { mas } x \notin W_{2,2} \\ \left(2, x_{l_{2}+2}, \ldots\right) \text { se } x \in W_{2,2} \\ T(x) \text { se } T(x) \in W_{0}\end{array}\right.$

(b) Para qualquer $x \in W_{2}, T(x) \in W_{0}$

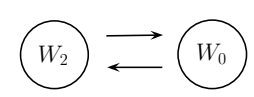


Definimos: $\tilde{T}(x)=\left\{\begin{array}{l}\left(2, x_{2}, x_{3}, x_{4}, \ldots\right) \text { se } x_{1}=0 \\ \left(0, x_{2}, x_{3}, x_{4}, \ldots\right) \text { se } x_{1}=2\end{array}\right.$ 


\section{CAPÍTULO 4}

\subsection{Caso particular}

Inicialmente faremos um caso particular no conjunto das sequências, ou seja, para esse caso, definimos $\tilde{T}: \Sigma_{N}^{+} \rightarrow \Sigma_{N}^{+}$próxima de $T$ com todas suas órbitas finalmente periódicas. Com isso, considerando um conjunto de Cantor, conseguimos generalizar o resultado para endomorfismos.

Considere $\Sigma_{N}^{+}$. Definimos:

$$
\begin{aligned}
& W_{1}=\left\{x: x=\left(1, x_{2}, x_{3}, \ldots\right) \in \Sigma_{N}^{+}\right\} \\
& W_{2}=\left\{x: x=\left(2, x_{2}, x_{3}, \ldots\right) \in \Sigma_{N}^{+}\right\} \\
& \vdots \\
& W_{i}=\left\{x: x=\left(i, x_{2}, x_{3}, \ldots\right) \in \Sigma_{N}^{+}\right\}
\end{aligned}
$$

Lema 4.1.1. Seja $T: \Sigma_{N}^{+} \rightarrow \Sigma_{N}^{+}$um endomorfismo tal que para todo $1 \leq i \leq N$, existe $x \in W_{i} \operatorname{com} T(x) \in W_{i}$.

Então, existe um endomorfismo $\tilde{T}: \Sigma_{N}^{+} \rightarrow \Sigma_{N}^{+}$tal que:

$$
\tilde{T}(x) \in W_{i} \Longleftrightarrow T(x) \in W_{i},
$$

e toda órbita de $\tilde{T}$ é finalmente periódica. 
Demonstração. Pela continuidade de $T$, para todo $i=1, \ldots, N$, existe $R_{i}=\overline{x_{2}}, \overline{x_{3}}, \ldots, \overline{x_{l_{i}}}$ com

$$
T\left(i, R_{i}, x_{l_{i}+1}, x_{l_{i}+2}, \ldots\right) \in W_{i}
$$

Observe que $l_{i}$ é tal que, se $(x)_{k}=(y)_{k}$ para $1 \leq k \leq l_{i}$ então $(T(x))_{1}=(T(y))_{1}$.

Consideremos para cada $i=1, \ldots, N$,

$$
W_{i, i}=\left\{x: x=\left(i, R_{i}, x_{l_{i}+1}, x_{l_{i}+2}, \ldots\right) \in \Sigma_{N}^{+}\right\} .
$$

Definimos:

$$
\tilde{T}(x)=\left\{\begin{array}{l}
\left(i, R_{i}, R_{i}, R_{i}, \ldots\right) \text { se } T(x) \in W_{i} \text { mas } x \notin W_{i, i} \\
\left(i, x_{l_{i}+2}, \ldots\right) \text { se } x \in W_{i, i}
\end{array}\right.
$$

Pela própria definição de $\tilde{T}$, temos que:

$$
\tilde{T}(x) \in K_{i} \Longleftrightarrow T(x) \in K_{i}
$$

Temos também que $\tilde{T}$ é endomorfismo pois, para todo $y=\left(i, y_{2}, y_{3}, \ldots\right)$, existe $x=\left(i, R_{i}, y_{2}, y_{3}, \ldots\right)$ tal que $\tilde{T}(x)=y$.

Mostremos que $\tilde{T}$ é finalmente periódica:

- Se $x \notin W_{i, i}$ e $T(x) \in W_{i}$ :

$$
\begin{aligned}
& \tilde{T}(x)=\left(i, R_{i}, R_{i}, \ldots\right) \in W_{i, i} \\
& \tilde{T}^{2}(x)=\left(i, R_{i}, R_{i}, \ldots\right)=\tilde{T}(x)
\end{aligned}
$$

- Se $x \in W_{i, i}$ e $\tilde{T}^{k}(x) \in W_{i, i}, \forall k \in \mathbb{N}$, então $x=\left(i, R_{i}, R_{i}, \ldots\right) \Rightarrow \tilde{T}(x)=x$.

- Se $x \in W_{i, i}$ e $\tilde{T}^{k}(x) \notin W_{i, i}$ para algum $k \in \mathbb{N}$, então, pelos casos anteriores, $\tilde{T}^{k+2}(x)=\tilde{T}^{k+1}(x)$.

Afim de utilizar esse resultado na resolução do nosso primeiro teorema, consideramos o seguinte lema: 
Lema 4.1.2. $W_{i}$ é um conjunto de Cantor para todo $i=1,2, \ldots, N$.

\section{Demonstração.}

i) $W_{i}$ é perfeito pois, tome $x=\left(i, \overline{x_{2}}, \overline{x_{3}}, \overline{x_{4}}, \ldots\right) \in W_{i}$ qualquer. Basta considerar a sequência dada por:

$$
\begin{aligned}
& x_{1}=(i, i, i, i, \ldots) \\
& x_{2}=\left(i, \overline{x_{2}}, \overline{x_{2}}, \overline{x_{2}}, \ldots\right) \\
& x_{3}=\left(i, \overline{x_{2}}, \overline{x_{3}}, \overline{x_{3}}, \overline{x_{3}}, \ldots\right) \\
& \vdots \\
& x_{n}=\left(i, \overline{x_{2}}, \overline{x_{3}}, \overline{x_{4}}, \ldots, \overline{x_{n}}, \overline{x_{n}}, \overline{x_{n}}, \ldots\right)
\end{aligned}
$$

Assim, $\left(x_{n}\right)$ é uma sequência em $W_{i}$ que converge para $x$, ou seja, $x$ é um ponto de acumulação.

ii) $W_{i}$ é fechado pois, tome $x=\left(\overline{x_{1}}, \overline{x_{2}}, \overline{x_{3}}, \ldots\right)$ um ponto de acumulação de $W_{i}$ qualquer. Sabemos que existe uma sequência $\left(x_{n}\right)$ em $W_{i}$ convergindo para $x$. Segue que, $\overline{x_{1}}=i$, ou seja, $x \in W_{i}$.

iii) $W_{i}$ é totalmente desconexo, pela definição.

\subsection{Resultados auxiliares}

Para auxiliar na demonstração dos teoremas principais, destacamos as seguintes proposições sobre a divisão de um conjunto de Cantor:

Proposição 4.2.1. Dados $\varepsilon>0$ e $K$ um conjunto de Cantor, existe $M>0$ e subconjuntos $K_{j} \subset K$ tais que:

i) $K_{j}$ é um conjunto de Cantor, com $0 \leq j \leq 2^{M}$;

ii) $K=\bigcup_{j=1}^{2^{M}} K_{j}$;

iii) Para cada $j=1,2,3, \ldots, 2^{M}$, $\operatorname{diam}\left(K_{j}\right)<\varepsilon$. 


\section{Demonstração.}

Por 2.1.5, sabemos que existe um homeomorfismo $h: K \rightarrow \tilde{K}$ entre quaisquer dois conjuntos de Cantor $K$ e $\tilde{K}$. Assim, para $\varepsilon>0$, existe $\delta>0$ tal que:

$$
d(x, y)<\delta \Rightarrow d\left(h^{-1}(x), h^{-1}(y)\right)<\varepsilon
$$

para quaisquer $x, y \in \tilde{K}$.

Sejam $\tilde{K}$ o conjunto ternário de Cantor e $M>0$ um inteiro tal que $\frac{1}{3^{M}}<\delta$.

Considere os intervalos $I(M, j)$ da construção do conjunto ternário de Cantor, com $1 \leq j \leq 2^{M}$, e tome:

$$
\tilde{K}_{j}=\bigcap_{n=M}^{\infty} I_{n} \bigcap I(M, j) \subset \tilde{K}
$$

Definimos $K_{j}=h^{-1}\left(\tilde{K}_{j}\right)$.

i) Para cada j, $\tilde{K}_{j}$ é um conjunto de Cantor pois, esse subconjunto de $\tilde{K}$ continua não contendo intervalos, todos seus pontos ainda são de acumulação e é uma interseção de fechados limitados na reta.

Segue do homeomorfismo $h$ que, $K_{j}$ é um conjunto de Cantor.

ii) Se $x \in \tilde{K}$ então $x \in I_{n}$ para $n \geq 1$, ou seja, $x \in I(M, j)$ para algum $j \in$ $\left\{1,2,3, \ldots, 2^{M}\right\}$.

Logo, $x \in \tilde{K}_{j}$ para algum $j \in\left\{1,2,3, \ldots, 2^{M}\right\}$, e portanto $\tilde{K}=\bigcup_{j=1}^{2^{M}} \tilde{K}_{j}$.

Segue do homeomorfismo $h$ que, $K=\bigcup_{j=1}^{2^{M}} K_{j}$.

iii) Para cada $j=1,2,3, \ldots, 2^{M}, \operatorname{diam}\left(\tilde{K}_{j}\right)=\frac{1}{3^{M}}<\delta$ pela própria construção do conjunto ternário de Cantor, e portanto, $\operatorname{diam}\left(K_{j}\right)<\varepsilon$.

Proposição 4.2.2. Dado K um conjunto de Cantor, existem uma sequência de subconjuntos $K_{m} \subset K$ e um ponto $p \in K$ tais que:

i) $K_{m}$ é um conjunto de Cantor, para qualquer $m \in \mathbb{N}$; 
ii) $K=\bigcup_{m=1}^{\infty} K_{m} \bigcup\{p\}$;

iii) Dado $\varepsilon>0$, existe $m_{0} \in \mathbb{N}$ tal que, para $m>m_{0}$, $\operatorname{diam}\left(K_{m}\right)<\varepsilon$.

\section{Demonstração.}

Por 2.1.5, sabemos que existe um homeomorfismo $h: K \rightarrow \tilde{K}$ entre dois conjuntos de Cantor $K$ e $\tilde{K}$. Assim, para $\varepsilon>0$, existe $\delta>0$ tal que:

$$
d(x, y)<\delta \Rightarrow d\left(h^{-1}(x), h^{-1}(y)\right)<\varepsilon
$$

para quaisquer $x, y \in \tilde{K}$.

Considere $\tilde{K}$ o conjunto ternário de Cantor e, para cada $m \in \mathbb{N}$, defina:

$$
\tilde{K}_{m}=\bigcap_{n=m}^{\infty} I_{n} \bigcap I\left(m, 2^{m}-1\right) \subset \tilde{K}
$$

onde $I_{n}$ e $I(n, j)$ são da construção do conjunto ternário de Cantor, com $1 \leq j \leq 2^{m}$ e $n \geq 1$.

Tome $K_{m}=h^{-1}\left(\tilde{K}_{m}\right)$.

i) Para cada m, $\tilde{K}_{m}$ é um conjunto de Cantor pois, esse subconjunto de $\tilde{K}$ continua não contendo intervalos, todos seus pontos ainda são de acumulação e é uma interseção de fechados limitados na reta.

Segue do homeomorfismo $h$ que, $K_{m}$ é um conjunto de Cantor.

ii) Seja $x \in \tilde{K}$ qualquer. Mostremos que $x \in \bigcup_{m=1}^{\infty} \tilde{K}_{m} \bigcup\{1\}$.

Temos que $x \in I_{n}$ para todo $n \geq 1$. Observe que:

Se $x \notin I(1,1)$ então $x \notin I(2,1)$ e $x \notin I(2,2)$. Segue que $x \in I(2,3)$ ou $x \in I(2,4)$.

Se além disso, $x \notin I(2,3)$ então $x \notin I(3,5)$ e $x \notin I(3,6)$. Segue que $x \in I(3,7)$ ou $x \in I(3,8)$.

De maneira geral, se $x \notin I\left(n, 2^{n}-1\right)$ para $n=1,2, \ldots m-1$ com $m \in \mathbb{N}$, então $x \notin I\left(m, 2^{m}-3\right)$ e $x \notin I\left(m, 2^{m}-2\right)$. Segue que $x \in I\left(m, 2^{m}-1\right)$ ou $x \in I\left(2,2^{m}\right)$.

Assim, $x \in I\left(m, 2^{m}-1\right)$ para algum $m \in \mathbb{N}$, ou $x \in I\left(m, 2^{m}\right)$ para todo $m \in \mathbb{N}$. Ou seja, $x \in \tilde{K}_{m}$ para algum $m \in \mathbb{N}$, ou $x=1$. 
Portanto, $\tilde{K}=\bigcup_{m=1}^{\infty} \tilde{K}_{m} \bigcup\{1\}$.

Segue do homeomorfismo $h$ que, $K=\bigcup_{m=1}^{\infty} K_{m} \bigcup\{p\}$.

iii) Dado $\varepsilon>0$, considere $m_{0} \in \mathbb{N}$ tal que $\frac{1}{3^{m_{0}}}<\delta$. Pela construção do conjunto ternário de Cantor, $\operatorname{diam}\left(\tilde{K}_{m_{0}}\right)=\frac{1}{3^{m_{0}}}<\delta$, e portanto, para $m>m_{0}, \operatorname{diam}\left(K_{m}\right)<\varepsilon$

Proposição 4.2.3. Um conjunto de Cantor pode ser dividido em $N$ conjuntos de Cantor.

\section{Demonstração.}

Como dois conjuntos de Cantor são homeomorfos (2.1.5), podemos considerar o conjunto ternário de Cantor $\tilde{K}$. Assim,

i) Se $N=2^{M}$ para algum inteiro $M>0$, para cada $1 \leq j \leq N$, considere o conjunto de Cantor:

$$
\tilde{K}_{j}=\bigcap_{n=M}^{\infty} I_{n} \bigcap I(M, j) \subset \tilde{K}
$$

onde os intervalos $I(M, j)$ são da construção do conjunto ternário de Cantor.

Analogamente as proposições anteriores, observamos que $\tilde{K}=\bigcup_{j=1}^{N} \tilde{K}_{j}$.

ii) Caso contrário, seja $M>0$ o menor inteiro tal que $N<2^{M}$. Para cada $1 \leq j \leq N$, considere o conjunto de Cantor:

$$
\tilde{K}_{j}=\bigcap_{n=M}^{\infty} I_{n} \bigcap I(M, j) \subset \tilde{K}
$$

onde os intervalos $I(M, j)$ são da construção do conjunto ternário de Cantor. Tome:

$$
\begin{aligned}
& K_{j}=\tilde{K}_{j}, \text { para } j=1,2, \ldots, N-1 ; \\
& K_{N}=\bigcup_{j=N}^{2^{M}} \tilde{K}_{j} .
\end{aligned}
$$


Assim, $\tilde{K}=\bigcup_{j=1}^{N} K_{j}$. Observe que $K_{N}$ também é um conjunto de Cantor pois é um subconjunto de $\tilde{K}$ que não contém intervalos, todos seus pontos são de acumulação e é uma união (disjunta) finita de fechados limitados na reta.

\subsection{Endomorfismos}

Teorema 4.3.1. Dados $T: K \rightarrow K$ um endomorfismo e $K_{1}, \ldots, K_{N}$ conjuntos de Cantor disjuntos com $K=\bigcup_{i=1}^{N} K_{i}$, existe um endomorfismo $\tilde{T}: K \rightarrow K$ tal que:

$$
\tilde{T}(x) \in K_{i} \Longleftrightarrow T(x) \in K_{i},
$$

e toda órbita de $\tilde{T}$ é finalmente periódica.

\section{Demonstração.}

A demonstração será feita por indução sobre $N$.

Para $\mathrm{N}=1$, defina o endomorfismo $\tilde{T}(x)=x$ para $x \in K_{1}=K$. Assim,

$$
\tilde{T}(x) \in K_{i} \Longleftrightarrow T(x) \in K_{i},
$$

e mais do que $\tilde{T}$ ser finalmente periódica, todos os seus pontos são fixos.

Suponha que que o teorema seja válido para $N-1$, e provemos que vale para $N$ qualquer.

$1^{o}$ Caso) Para todo $i=1, \ldots, N$, existe $x \in K_{i}$ tal que $T(x) \in K_{i}$

Por 4.1.2, cada $K_{i}$ é homeomeorfo a $W_{i}=\left\{\left(i, x_{2}, x_{3}, \ldots\right) \in \Sigma_{N}^{+}\right\}$. Assim, para $N$ qualquer, por 4.1.1, existe um endomorfismo $\tilde{T}: K \rightarrow K$ tal que,

$$
\tilde{T}(x) \in K_{i} \Longleftrightarrow T(x) \in K_{i},
$$


e toda órbita de $\tilde{T}$ é finalmente periódica.

$2^{o}$ Caso) Existe algum j, com $1 \leq j \leq N$ tal que, para todo $x \in K_{j}$ temos que $T(x) \notin K_{j}$.

Denotamos:

$$
W_{S_{l}}=\left\{x \in K_{j}: T(x) \in K_{S_{l}}\right\} \neq \emptyset
$$

$\operatorname{com} S_{l} \neq j$ e $l=1, \ldots, m$, para algum $1 \leq m \leq N$.

$$
U_{P_{q}}=\left\{x \in K_{P_{q}}: T(x) \in K_{j}\right\} \neq \emptyset
$$

$\operatorname{com} P_{q} \neq j$ e $q=1, \ldots, n$, para algum $1 \leq n \leq N$

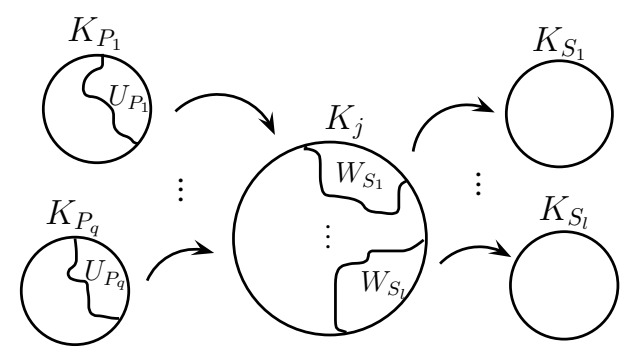

Mostremos que os conjuntos $W_{S_{l}}$ e $U_{P_{q}}$ são conjuntos de Cantor.

i) $W_{S_{l}}=T^{-1}\left(K_{S_{l}}\right) \cap K_{j}$ é fechado pois, $T$ é contínua e $K_{S_{l}}, K_{j}$ são fechados.

ii) Seja $x \in W_{S_{l}} \subset K_{j}$. Como $K_{j}$ é perfeito, existe uma sequência $x_{n} \in K_{j}$ com $x_{n} \rightarrow x$. Verificaremos que existe $n_{0}$ tal que, para $n>n_{o}, x_{n} \in W_{S_{l}}$.

Observe que $K_{S_{l}}$ é aberto, pois $\left(K_{S_{l}}\right)^{c}=K_{1} \cup K_{2} \cup \ldots \cup K_{S_{l-1}} \cup K_{S_{l+1}} \cup \ldots \cup K_{N}$ é uma união finita de fechados. Pela continuidade de $T, T^{-1}\left(K_{S_{l}}\right)$ é aberto. Segue que $x \in T^{-1}\left(K_{S_{l}}\right)$ admite uma vizinhança $V(x) \subset K_{j}$ totalmente contida em $T^{-1}\left(K_{S_{l}}\right)$, ou seja, $V(x) \subset W_{S_{l}}$. Assim, para algum $n_{0}$, se $n>n_{0}, V(x)$ contém $x_{n}$.

Logo, $W_{S_{l}}$ é perfeito.

iii) Temos que $K_{j}$ é totalmente desconexo, ou seja, cada uma de suas componentes conexas é um ponto. Como $W_{S_{l}}$ é subconjunto de $K_{j}$, também tem essa propriedade.

Portanto, $W_{S_{l}}$ é totalmente desconexo. 
Por i), ii) e iii), $W_{S_{l}}$ é um conjunto de Cantor. Analogamente, $U_{P_{q}}$ também é.

Pela proposição 4.2 .3 , segue que os conjuntos $U_{P_{q}}$ e $W_{S_{l}}$ podem ser subdivididos em conjuntos de Cantor. Sem perda de generalidade, suponha $m<n$. Divida $W_{S_{m}}$ em $n-m+1$ conjuntos de Cantor não vazios $\overline{W_{S_{l}}} \operatorname{com} m \leq l \leq n$. Por abuso de notação, denotaremos por $W_{S_{l}}$, com $1 \leq l \leq n$.

Por 2.1.5, temos que para cada $l=1, \ldots, n$ existe um homeomorfismo $h_{l}: U_{P_{l}} \rightarrow W_{S_{l}}$.

Definimos $\hat{T}: K \rightarrow K$ por:

$$
\hat{T}(x)=\left\{\begin{array}{l}
h_{l}(x) \text { se } x \in U_{P_{l}}, l=1, \ldots, n \\
T(x) \text { se } x \notin U_{P_{l}}, l=1, \ldots, n
\end{array}\right.
$$

Observe que $\hat{T}$ é um endomorfismo que satisfaz:

$$
\hat{T}(x) \in K_{i} \Longleftrightarrow T(x) \in K_{i}
$$

Além disso, $\left.\hat{T}\right|_{\bigcup_{l=1}^{n} U_{P_{l}}}$ é um homeomorfismo sobre sua imagem $K_{j}$.

Definimos também o endomorfismo $g: K \backslash K_{j} \rightarrow K \backslash K_{j}$ por:

$$
g(x)=\left\{\begin{array}{l}
T(x) \text { se } x \notin U_{P_{l}}, l=1, \ldots, n \\
\hat{T}^{2}(x) \text { se } x \in U_{P_{l}}, l=1, \ldots, n
\end{array}\right.
$$

Pela hipótese de indução, existe um endomorfismo $\tilde{g}: K \backslash K_{j} \rightarrow K \backslash K_{j}$ tal que para $i=1, \ldots, j-1, j+1, \ldots, N$ :

$$
\tilde{g}(x) \in K_{i} \Longleftrightarrow g(x) \in K_{i}
$$

e toda órbita é finalmente periódica.

Finalmente, tome $\tilde{T}: K \rightarrow K$ definida por:

$$
\tilde{T}(x)= \begin{cases}\tilde{g}(x) & \text { se } x \notin U_{P_{l}} \text { e se } x \notin K_{j}, l=1, \ldots, n \\ \hat{T}(x) & \text { se } x \in U_{P_{l}}, l=1, \ldots, n \\ \tilde{g}\left(h_{l}^{-1}(x)\right) & \text { se } x \in K_{j}\end{cases}
$$

$\tilde{T}$ é um endomorfismo tal que:

$$
\tilde{T}(x) \in K_{i} \Longleftrightarrow T(x) \in K_{i},
$$


e toda órbita é finalmente periódica, como queríamos.

Teorema (Endomorfismos). Seja K um conjunto de Cantor. Dados um endomorfismo $T: K \rightarrow K$ e $\varepsilon>0$, existe um endomorfismo $\tilde{T}: K \rightarrow K$ tal que:

$$
D(T, \tilde{T})=\max _{x \in K} d(T(x), \tilde{T}(x))<\varepsilon
$$

e toda órbita de $\tilde{T}$ é finalmente periódica.

Demonstração. Por 4.2.1, existem $K_{1}, K_{2}, \ldots, K_{2^{M}}$ conjuntos de Cantor lineraes disjuntos, com $K=\bigcup_{j=1}^{2^{M}}$, e $\operatorname{diam}\left(K_{j}\right)<\varepsilon$ para $j=1,2, \ldots, 2^{M}$.

Pelo teorema anterior 4.3.1, existe um endomorfismo $\tilde{T}: K \rightarrow K$ tal que:

$$
\tilde{T}(x) \in K_{i} \Longleftrightarrow T(x) \in K_{i},
$$

e toda órbita é finalmente periódica.

Ou seja, existe um endomorfismo $\tilde{T}: K \rightarrow K$ tal que:

$$
D(T, \tilde{T})=\max _{x \in K} d(T(x), \tilde{T}(x))<\varepsilon
$$

e toda órbita de $\tilde{T}$ é finalmente periódica.

\subsection{Homeomorfismos}

Lema 4.4.1. Sejam $T: K \rightarrow K$ um homeomorfismo e $K_{1}, K_{2}, \ldots, K_{N}$ conjuntos de Cantor disjuntos com $K=\bigcup_{i=1}^{N} K_{i}$. Considere que para cada $1 \leq i \leq N$, uma das seguintes propriedades é satisfeita:

i) Para qualquer $y \in K_{i}$ temos que $T^{-1}(y) \in K_{i}$ 


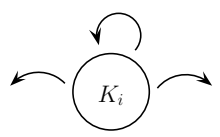

ii) Para qualquer $x \in K_{i}$ temos que $T(x) \in K_{i}$

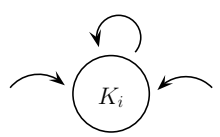

Então, existe um homeomorfismo $\tilde{T}: K \rightarrow K$ tal que:

$$
\tilde{T}(x) \in K_{i} \Longleftrightarrow T(x) \in K_{i},
$$

e toda órbita de $\tilde{T}$ converge para um ponto fixo.

\section{Demonstração.}

Fixemos a notação $K_{R_{l}}$ para os conjuntos de Cantor que satisfazem a propriedade i), com $l=1, \ldots, r$ para algum $1 \leq r \leq N$. E, para os conjuntos de Cantor que satisfazem a propriedade ii), fixemos a notação $K_{A_{q}}$, com $q=1, \ldots, n$ para algum $1 \leq n \leq N$. Note que, $N=q+r$.

i) Para cada $K_{R_{l}}$, com $l=1, \ldots, r$ para algum $1 \leq r \leq N$, denotamos:

$$
W_{A_{q}}^{R_{l}}=\left\{x \in K_{R_{l}}: T(x) \in K_{A_{q}}\right\} \neq \emptyset
$$

$\operatorname{com} q=1, \ldots, n$, para algum $1 \leq n \leq N . \mathrm{E}$

$$
V_{R_{l}}=\left\{x \in K_{R_{l}}: T(x) \in K_{R_{l}}\right\} .
$$

Como já visto em 4.3.1, os conjuntos $W_{A_{q}}^{R_{l}}$ são conjuntos de Cantor. Analogamente, $V_{R_{l}}$ também é um conjunto de Cantor.

Pela proposição 4.2 .2 , para cada $l=1, \ldots, r$ com $1 \leq r \leq N$, existe uma sequência de conjuntos de Cantor $\tilde{K}_{m} \subset V_{R_{l}}$ e um ponto $p \in V_{R_{l}}$ tais que, $V_{R_{l}}=\bigcup_{m=1}^{\infty} \tilde{K}_{m} \bigcup\{p\}$ $\operatorname{com} \operatorname{diam}\left(\tilde{K}_{m}\right) \rightarrow 0$ 


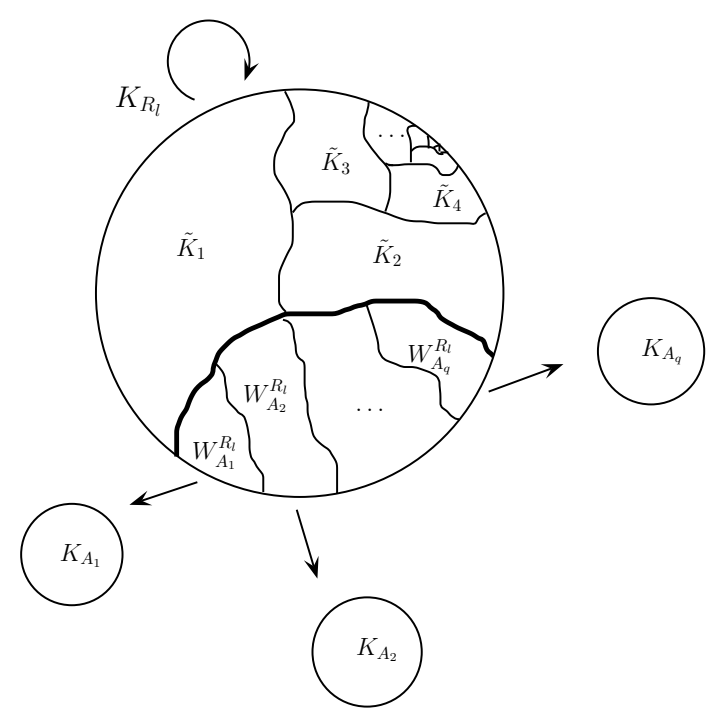

Por 2.1.5, temos que para cada $m \geq 2$, existe um homeomorfismo $h_{m}: \tilde{K}_{m} \rightarrow \tilde{K}_{m-1}$. Temos também que, como $W_{A_{1}}^{R_{l}} \cup W A_{2}{ }^{R_{l}} \cup \ldots \cup W_{A_{q}}^{R_{l}}$ é um conjunto de Cantor, existe um homeomorfismo $\bar{h}: \tilde{K}_{1} \rightarrow W_{A_{1}}^{R_{l}} \cup W_{A_{2}}^{R_{l}} \cup \ldots \cup W_{A_{q}}^{R_{l}}$, $\operatorname{com} l=1, \ldots, r$ e $1 \leq r \leq N$.

Definimos $\hat{T_{R_{l}}}: K_{R_{l}} \rightarrow K_{R_{l}}$ por:

$$
\hat{T_{R_{l}}}(x)= \begin{cases}h_{m}(x) & \text { se } x \in \tilde{K}_{m}, m \geq 2 \\ \bar{h}(x) & \text { se } x \in \tilde{K}_{1} \\ p & \text { se } x=p\end{cases}
$$

Temos que, ${\hat{T_{R_{l}}}}^{m+1}(x) \notin V_{R_{l}}$, para $x \in \tilde{K}_{m} \operatorname{com} x \neq p$.

Observe que para cada $K_{R_{l}}$, a sequência $\tilde{K}_{m}$, o ponto $p$ e os homeomorfismos $h_{m}$ também deveriam ser indexados por $R_{l}$, mas não faremos isso para não carregar a notação. Assim, quando esses forem citados, indicaremos qual o conjunto de Cantor referente.

ii) Para cada $K_{A_{q}}, \operatorname{com} q=1, \ldots, n$ para algum $1 \leq n \leq N$, sabemos da proposição 4.2.2 que, existe uma sequência de conjuntos de Cantor $\tilde{K}_{m} \subset \tilde{K}_{A_{q}}$ e um ponto $p \in K_{A_{q}}$ tais que, $K_{A_{q}}=\bigcup_{m=1}^{\infty} \tilde{K}_{m} \bigcup\{p\} \operatorname{com} \operatorname{diam}\left(\tilde{K}_{m}\right) \rightarrow 0$.

Por 2.1.5, temos que para cada $m \geq 2$, existe um homeomorfismo $h_{m}: \tilde{K}_{m-1} \rightarrow \tilde{K}_{m}$ 
Definimos $\hat{T_{A_{q}}}: K_{A_{q}} \rightarrow K_{A_{q}}$ por:

$$
\hat{T_{A_{q}}}(x)= \begin{cases}h_{m}(x) & \text { se } x \in \tilde{K}_{m-1}, m \geq 2 \\ p & \text { se } x=p\end{cases}
$$

Temos que, $\hat{T}_{A_{q}}(x)$ converge para o ponto fixo $p \in K_{A_{q}}$, para qualquer $x \in K_{A_{q}}$.

Observe que aqui também, para cada $K_{A_{q}}$, a sequência $\tilde{K}_{m}$, o ponto $p$ e os homeomorfismos $h_{m}$ deveriam ser indexados por $A_{q}$.

Afim de definir o homeomorfismo em $K$, por 2.1.5, usamos que, para cada $A_{q}$ com $q=1, \ldots, n$ e $1 \leq n \leq N$, existe um homeomorfismo $h_{A_{q}}: W_{A_{q}}^{R_{1}} \cup W_{A_{q}}^{R_{2}} \cup \ldots \cup W_{A_{q}}^{R_{l}} \rightarrow \tilde{K}_{1} \subset K_{A_{q}}$.

Logo, considerando i) e ii), podemos definir $\tilde{T}: K \rightarrow K$ por:

$$
\tilde{T}(x)= \begin{cases}\hat{T_{R_{l}}}(x) & \text { se } x \in V_{R_{l}}, l=1, \ldots, r \\ \hat{T_{A_{q}}}(x) & \text { se } x \in K_{A_{q}}, q=1, \ldots, n \\ h_{A_{q}}(x) & \text { se } x \in K_{R_{l}} \backslash V_{R_{l}}, l=1, \ldots, r\end{cases}
$$

Assim, $\tilde{T}$ é um homeomorfismo que satisfaz:

$$
\tilde{T}(x) \in K_{i} \Longleftrightarrow T(x) \in K_{i},
$$

para todo $i=1, \ldots, N$. Além disso, toda órbita de $\tilde{T}$ converge para o ponto fixo de $\operatorname{algum} K_{A_{q}}, \operatorname{com} q=1, \ldots, n$ e $1 \leq n \leq N$.

Particularmente, podemos ter as propriedades i) e ii) sendo satisfeitas para algum $K_{i} \operatorname{com} 1 \leq i \leq N$ :

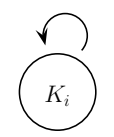

Neste caso, podemos definir $\tilde{T}(x)=x$ para qualquer $x \in K_{i}$. Teremos que, mais do que toda órbita de $\tilde{T}$ convergir para um ponto fixo, todos os pontos de $K_{i}$ são fixos.

Teorema 4.4.2. Dados $T: K \rightarrow K$ um homeomorfismo e $K_{1}, \ldots, K_{N}$ conjuntos de 
Cantor disjuntos com $K=\bigcup_{i=1}^{N} K_{i}$, existe um homeomorfismo $\tilde{T}: K \rightarrow K$ tal que:

$$
\tilde{T}(x) \in K_{i} \Longleftrightarrow T(x) \in K_{i},
$$

e o w-limite de toda órbita de $\tilde{T}$ é uma órbita periódica.

\section{Demonstração.}

A demonstração será feita por indução sobre $N$.

Para $\mathrm{N}=1$, defina o homeomorfismo $\tilde{T}(x)=x$ para $x \in K_{1}=K$. Assim,

$$
\tilde{T}(x) \in K_{i} \Longleftrightarrow T(x) \in K_{i},
$$

e mais do que o $w$-limite de toda órbita de $\tilde{T}$ ser uma órbita periódica., $\tilde{T}$ tem todos os seus pontos fixos.

Suponha que que o teorema seja válido para $N-1$, e provemos que vale para $N$ qualquer.

$1^{\circ}$ Caso) Para todo $i=1, \ldots, N$ temos que $K_{i}$ satisfaz uma das seguintes propriedades:

i) Para qualquer $y \in K_{i}$ temos que $T^{-1}(y) \in K_{i}$

ii) Para qualquer $x \in K_{i}$ temos que $T(x) \in K_{i}$

Por 4.4.1, sabemos que para qualquer $N$, existe um homeomorfismo $\tilde{T}: K \rightarrow K$ tal que:

$$
\tilde{T}(x) \in K_{i} \Longleftrightarrow T(x) \in K_{i},
$$

e toda órbita de $\tilde{T}$ converge para um ponto fixo. 
$2^{o}$ Caso) Para algum $j \in\{1,2, \ldots, N\}$, existe $x \in K_{j}$ tal que $T(x) \notin K_{j}$ e existe $y \in K_{j}$ tal que $T^{-1}(y) \notin K_{j}$

Denotamos:

$$
W_{S_{l}}=\left\{x \in K_{j}: T(x) \in K_{S_{l}}\right\} \neq \emptyset
$$

com $S_{l} \neq j$ e $l=1, \ldots, m$, para algum $1 \leq m \leq N$.

$$
U_{P_{q}}=\left\{x \in K_{P_{q}}: T(x) \in K_{j}\right\} \neq \emptyset,
$$

com $P_{q} \neq j$ e $q=1, \ldots, n$, para algum $1 \leq n \leq N$. E

$$
V_{j}=\left\{x \in K_{j}: T(x) \in K_{j}\right\} .
$$

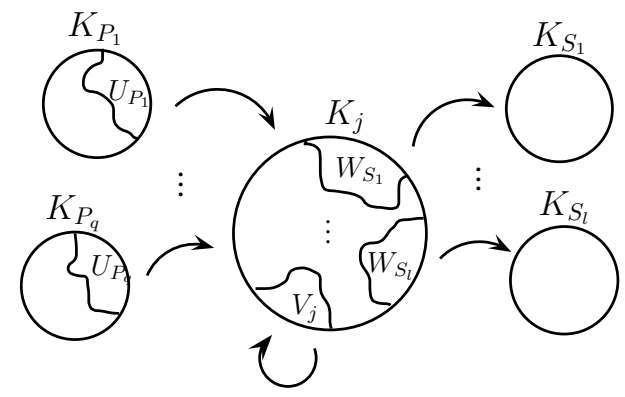

Como já visto em 4.3.1, os conjuntos $W_{S_{l}}$ e $U_{P_{q}}$ são conjuntos de Cantor.

Pela proposição 4.2.3, segue que os conjuntos $U_{P_{q}}$ e $W_{S_{l}}$ podem ser subdivididos em conjuntos de Cantor. Sem perda de generalidade, suponha $m<n$. Divida $W_{S_{m}}$ em $n-m+1$ conjuntos de Cantor não vazios $\overline{W_{S_{l}}} \operatorname{com} m \leq l \leq n$. Por abuso de notação, denotaremos por $W_{S_{l}}$, com $1 \leq l \leq n$.

Por 2.1.5, temos que para cada $l=1, \ldots, n$ existe um homeomorfismo $h_{l}: U_{P_{l}} \rightarrow W_{S_{l}}$.

Definimos $\hat{T}: K \rightarrow K$ por:

$$
\hat{T}(x)=\left\{\begin{array}{l}
h_{l}(x) \text { se } x \in U_{P_{l}}, l=1, \ldots, n \\
T(x) \text { se } x \notin U_{P_{l}}, l=1, \ldots, n
\end{array}\right.
$$

Observe que $\hat{T}$ é um homeomorfismo que satisfaz:

$$
\hat{T}(x) \in K_{i} \Longleftrightarrow T(x) \in K_{i} .
$$


Além disso, $\left.\hat{T}\right|_{\bigcup_{l=1}^{n} U_{P_{l}}}$ é um homeomorfismo sobre sua imagem $K_{j}$.

Definimos também o homeomorfismo $g: K \backslash K_{j} \rightarrow K \backslash K_{j}$ por:

$$
g(x)=\left\{\begin{array}{l}
T(x) \text { se } x \notin U_{P_{l}}, l=1, \ldots, n \\
\hat{T}^{2}(x) \text { se } x \in U_{P_{l}}, l=1, \ldots, n
\end{array}\right.
$$

Pela hipótese de indução, existe um homeomorfismo $\tilde{g}: K \backslash K_{j} \rightarrow K \backslash K_{j}$ tal que para $i=1, \ldots, j-1, j+1, \ldots, N$ :

$$
\tilde{T}(x) \in K_{i} \Longleftrightarrow T(x) \in K_{i},
$$

e toda órbita converge para um ponto fixo.

Finalmente, tome $\tilde{T}: K \rightarrow K$ definida por:

$$
\tilde{T}(x)= \begin{cases}\tilde{g}(x) & \text { se } x \notin U_{P_{l}} \text { e se } x \notin K_{j}, l=1, \ldots, n \\ \hat{T}(x) & \text { se } x \in U_{P_{l}}, l=1, \ldots, n \\ \tilde{g}\left(\hat{T}^{-1}(x)\right) & \text { se } x \in K_{j} \backslash V_{j} \\ x & \text { se } x \in V_{j}\end{cases}
$$

$\tilde{T}$ é um homeomorfismo tal que:

$$
\tilde{T}(x) \in K_{i} \Longleftrightarrow T(x) \in K_{i},
$$

e o $w$-limite de toda órbita de $\tilde{T}$ é uma órbita periódica., como queríamos.

Teorema (Homeomorfismos). Seja K um conjunto de Cantor. Dados um homeomorfismo $T: K \rightarrow K$ e $\varepsilon>0$, existe um homeomorfismo $\tilde{T}: K \rightarrow K$ tal que:

$$
D(T, \tilde{T})=\max _{x \in K} d(T(x), \tilde{T}(x))<\varepsilon
$$

e o w-limite de toda órbita de T̃ é uma órbita periódica.

Demonstração. Por 4.2.1, existem $K_{1}, K_{2}, \ldots, K_{2^{M}}$ conjuntos de Cantor lineraes disjuntos, com $K=\bigcup_{j=1}^{2^{M}}$, e $\operatorname{diam}\left(K_{j}\right)<\varepsilon$ para $j=1,2, \ldots, 2^{M}$. 
Pelo teorema anterior 4.4.2, existe um homeomorfismo $\tilde{T}: K \rightarrow K$ tal que:

$$
\tilde{T}(x) \in K_{i} \Longleftrightarrow T(x) \in K_{i},
$$

e o $w$-limite de toda órbita de $\tilde{T}$ é uma órbita periódica.

Ou seja, existe um homeomorfismo $\tilde{T}: K \rightarrow K$ tal que:

$$
D(T, \tilde{T})=\max _{x \in K} d(T(x), \tilde{T}(x))<\varepsilon
$$

e o $w$-limite de toda órbita de $\tilde{T}$ é uma órbita periódica. 

[1] Bernardes Jr., N. C., Darji, U. B., Graph theoretic structure of maps of the Cantor space, Advances in Mathematics, 231 (2012), 1655-1680.

[2] Bezuglyi, S., Dooley, A. H. e Kwiatkowski, J., Topologies on the group of Borel automorphisms of a standard Borel space, Topol. Methods in Nonlinear Anal., 27 (2006), 333-385.

[3] Bezuglyi, S., Dooley, A. H. e Kwiatkowski, J., Topologies on the group of Borel homeomorphisms of a Cantor set, Topol. Methods in Nonlinear Anal., 27 (2006), 299-331.

[4] Bezuglyi, S., Kwiatkowski, J. e Medynets, K., Aproximation in measurable Borel, and Cantor dynamics, Contemp. Math. Americ. Math. Society, 385 (2005), 39-64.

[5] Bissacot, R. e Freire Jr, R. S., On the existence of maximizing measures for irreducible countable Markov shifts: a dynamical proof, Ergodic Theory and Dynamical Systems, 1 (2013), 1-13.

[6] Bousch, T. e Jenkinson, O., Cohomology classes of dynamically non-negative $C^{k}$ functions, Inventiones Mathematicae, 148 (2002), 207-217. 
[7] Bousch, T., La condition de Walters, Ann. Scientifiques de l'Ecole Normale Supérieure, 34 (2001), 287-311.

[8] Bousch, T., Le poisson n'a pas d'arêtes, Ann. Inst. Henri Poincaré (Proba. et Stat.), 36 (2000), 489-508.

[9] Brémont, J., Entropy and maximizing measures of generic continuous functions, C. R. Math. Acad. Sci., 346 (2008), 199-201.

[10] Contreras, G., Lopes, A. e Thieullen, P., Maximizing measures for expanding transformations, arXiv:1307.0533 [math.DS], (2013).

[11] Faria, E., Dimensão fracionária e conjuntos de Cantor, Dissertação de mestrado $U S P,(1985)$.

[12] Gonschorowski, J. S., Densidade do conjunto de endomorfismos com medida maximizante suportada em órbita periódica, Tese de doutorado USP, (2012).

[13] Halmos, P., Approximation theories for measure-preserving transformations, Transactions of the American Mathematical Society, 55 (1944), 1-18.

[14] Jenkinson, O., Mauldin, R. D. e Urbański, M., Ergodic optimization for noncompact dynamical systems, Dynamical Systems, 22 (2007), 379-388.

[15] Jenkinson, O., Mauldin, R. D. e Urbański, M., Ergodic optimization for countable alphabet subshifts of finite type, Ergodic Theory and Dynamical Systems, $\mathbf{2 6}$ (2006), 1791-1803.

[16] Jenkinson, O., Ergodic optimization, Discrete and Continuous Dynamical Systems, 15 (2006), 197-224.

[17] Jenkinson, O., Every ergodic measure is uniquely maximizing, Discrete and Continuous Dynamical Systems, 16 (2006), 383-392.

[18] Mañé, R., Introdução à teoria ergódica, Rio de Janeiro, Impa (1983). 
[19] Medynets, K., On approximation of homeomorphisms of a Cantor set, Fundamenta Mathematicae, 194 (2007), 1-13.

[20] Morris, I. D., Maximizing measures of generic Hölder functions have zero entropy, Nonlinearity, 21 (2008), 993-1000.

[21] Murdeshear, M. B., General topology, New York, John Wiley \& Sons (1983).

[22] Rokhlin, V., Selected topics from the metric theory of dynamical systems, Americ. Math. Soc. Translations, 49 (1966), 171-240.

[23] Sears, M., Expansive self-homeomorphisms of the Cantor set, Math. Systems Theory, 6 (1972), 129-132.

[24] Shimomura, T., The pseudo-orbit tracing property and expansiveness on the Cantor set, Americ. Math. Society, 106 (1989), 241-244.

[25] Sierpinski, W., General topology, Toronto, University of Toronto Press (Mathematical Expositions, $\left.N^{0}=7\right)(1952)$.

[26] Tal, F. e Addas-Zanata, S., Maximizing measures for endomorphisms of the circle, Nonlinearity, 21 (2008), 2347-2359.

[27] Tal, F. e Addas-Zanata, S., On maximizing measures of homeomorphisms on compact manifolds, Fundamenta Mathematicae, 200 (2008), 145-159.

[28] Tal, F. e Addas-Zanata, S., Support of maximizing measures for typical $C^{0}$ dynamics on compact manifolds, Discrete and Continuous Dynamical Systems, 26 (2010), 795-804.

[29] Walters, P., An introduction to Ergodic Theory, New York, Springer-Verlag (1982). 